\title{
Research on Feature Extraction of Performance Degradation for Flexible Material R2R Processing Roller Based on PCA
}

\author{
Yaohua Deng $\left(\mathbb{D}\right.$, Huiqiao Zhou $\left(\mathbb{D}\right.$, Kexing Yao $\mathbb{D}$, Zhiqi Huang $\mathbb{D}^{\mathbb{D}}$, and Chengwang Guo \\ School of Electromechanical Engineering, Guangdong University of Technology, Guangzhou, Guangdong, China \\ Correspondence should be addressed to Zhiqi Huang; hzq2111801162@mail2.gdut.edu.cn
}

Received 16 June 2020; Revised 29 November 2020; Accepted 11 December 2020; Published 28 December 2020

Academic Editor: Bhupendra Khandelwal

Copyright (C) 2020 Yaohua Deng et al. This is an open access article distributed under the Creative Commons Attribution License, which permits unrestricted use, distribution, and reproduction in any medium, provided the original work is properly cited.

\begin{abstract}
Performance feature extraction is the primary problem in equipment performance degradation assessment. To handle the problem of high-dimensional performance characterization and complexity of calculating the performance indicators in flexible material roll-to-roll processing, this paper proposes a PCA method for extracting the degradation characteristic of roll shaft. Based on the analysis of the performance influencing factors of flexible material roll-to-roll processing roller, a principal component analysis extraction model was constructed. The original feature parameter matrix composed of 10-dimensional feature parameters such as time domain, frequency domain, and time-frequency domain vibration signal of the roll shaft was established; then, we obtained a new feature parameter matrix $Z_{\text {org }}{ }^{*}$ by normalizing the original feature parameter matrix. The correlation measure between every two parameters in the matrix $Z_{\text {org }}{ }^{*}$ was used as the eigenvalue to establish the covariance matrix of the performance degradation feature parameters. The Jacobi iteration method was introduced to derive the algorithm for solving eigenvalue and eigenvector of the covariance matrix. Finally, using the eigenvalue cumulative contribution rate as the screening rule, we linearly weighted and fused the eigenvectors and derived the feature principal component matrix $F$ of the processing roller vibration signal. Experiments showed that the initially obtained, 10-dimensional features of the processing rollers' vibration signals, such as average, root mean square, kurtosis index, centroid frequency, root mean square of frequency, standard deviation of frequency, and energy of the intrinsic mode function component, can be expressed by 3-dimensional principal components $F_{1}, F_{2}$, and $F_{3}$. The vibration signal features reduction dimension was realized, and $F_{1}, F_{2}$, and $F_{3}$ contain $98.9 \%$ of the original vibration signal data, further illustrating that the method has high precision in feature parameters' extraction and the advantage of eliminating the correlation between feature parameters and reducing the workload selecting feature parameters.
\end{abstract}

\section{Introduction}

The object of flexible material roll-to-roll processing is flexible electronic film material; once the core components of processing equipment begin to degrade, flexible electronic film products will be deformed to varying degrees, resulting in processing quality problems $[1,2]$. Therefore, it is necessary to predict the performance and health status of the core components of flexible material R2R processing equipment. However, the number of rollers of the flexible material R2R processing equipment is large, and there are correlations between the movement state variables of the collected rollers, which lead to complications in the process of extracting performance degradation feature. If the performance degradation feature parameters of each roller are analyzed separately, the results are often isolated; if the parameters are blindly reduced, it may lead to incorrect performance state prediction conclusions due to the loss of too much raw information. Thus, it is extremely necessary to fuse and reduce the dimensions of the feature data for roll shaft performance degradation prediction and extract representative feature [3].

Previous studies have shown that principal component analysis (PCA), based on the idea of spatial 
transformation, achieves the purpose of optimal variance without reducing the information content contained in the original data and describes the high-dimensional data information with less principal component information, which has incomparable advantages over other algorithms [4-6]. In reference [4] (2018), spearman grade correlation coefficient and PCA were used for feature fusion to obtain the health index representing the declining state of rolling bearing performance. The analysis results of an example show that the proposed method can accurately identify the declining state of the rolling bearing performance. In reference [5] (2018), a frequency analysis method based on the residuals generated by SOM-PCA algorithm is proposed. This method is effective for fault detection and diagnosis of most of the unsupervised classification experimental data sets. In reference [6] (2018) proposed a sensor fault detection method of water chiller sensor based on empirical mode decomposition (EMD) threshold denoising and principal component analysis (EMD-TD-PCA). PCA model is established by EMD threshold denoising data. $Q$ statistic is used to detect sensor fault. Compared with the traditional PCA method, EMD-TD-PCA method can effectively improve the efficiency of fault detection. Therefore, on the basis of previous research, this paper proposes a PCA method for extracting the degradation feature of $\mathrm{R} 2 \mathrm{R}$ roll shaft of flexible material, constructs the principal component analysis extraction model of the degradation feature of processing roll shaft, establishes the feature matrix of the original vibration signal of roll shaft and the covariance matrix of the degradation feature, and introduces Jacobi method. The eigenvalues and eigenvectors of the covariance matrix, as well as the principal component matrix algorithm of the vibration signal of the processing roll, are studied. Finally, the validity and effectiveness of the proposed method for extracting the performance degradation eigenvalues are verified by $R 2 R$ processing experiments.

\section{Flexible Material R2R Processing Roller Performance Degradation Feature Extraction Based on PCA}

The principle framework of flexible material R2R performance degradation feature extraction is shown in Figure 1. As shown in Figure 1, the vibration acceleration data of roller axle during R2R processing were collected by sensors. After filtering and denoising the vibration acceleration signal data, the eigenvalues of the vibration acceleration data in the time domain, frequency domain, and time-frequency domain were calculated by integrating mathematical statistics, power spectrum, and empirical mode decomposition (EMD) [7-9]. On this basis, the eigenvalue normalized feature matrix and the covariance matrix of eigenvalue were established. Finally, using the eigenvalue cumulative contribution rate as the screening rule, we linearly weighted and fused the eigenvectors, then obtained the degradation feature parameters principal component of processing roll shaft performance, i.e., the $\mathrm{R} 2 \mathrm{R}$ processing roller shaft performance degradation indicator characterizing amount.

\section{Algorithm Derivation of PCA Extraction Model of Processing Roller's Performance Degradation Feature}

Considering the correlations between the vibration signal features of the unwinding roller, the guide roller, the driving roller, and the rewinding roller of R2R processing equipment, the principal component analysis (PCA) method is introduced to study the weighted fusion and dimensionality reduction of processing roller vibration feature data, i.e., the extraction of the feature parameters of roller performance degradation [10].

3.1. Establishing the Matrix of Original Vibration Signal of Feature Parameters. Time-domain analysis and frequencydomain analysis are easy to calculate, and physical meaning of their feature parameters is clear. The time-frequency eigenvalues of the original vibration signal of R2R roller are listed as follows. The original vibration signal of roller in the flexible material processing system is represented by $x$. The sample length is $L$, the sliding window width is $M$, and the sample mean value of smooth filter output is $y_{L}$. According to the definition of sliding average filtering [11], $y_{L}$ can be obtained as:

$$
y_{L}=\frac{x_{L}+x_{L-1}+x_{L-2}+\cdots+x_{L-M+1}}{M}
$$

Based on the data after smoothing filtering, matrices of primitive feature parameters of processing roll axis vibration signal that were analyzed in the time domain, frequency domain, and time-frequency domain can be established.

We postulated the number of samples is $n$, and feature parameter value of roller vibration signal is $Z_{\mathrm{ij}}\left(Z_{\mathrm{ij}}\right.$ represents the No $j$ feature variable value of the No i sample data), so the feature parameter matrix of roller vibration signal was expressed as follows:

$$
Z=\left(\begin{array}{cccc}
z_{11} & z_{12} & \cdots & z_{1 m} \\
z_{21} & z_{\mathrm{ij}} & \cdots & z_{2 m} \\
\vdots & \vdots & \cdots & \vdots \\
z_{n 1} & z_{n 2} & \cdots & z_{n m}
\end{array}\right)=\left(Z_{1}, Z_{2}, \cdots, Z_{m}\right)
$$

In equation (2), $Z=\left(Z_{1}, Z_{2}, \cdots, Z_{m}\right)$ is a simplified expression of the feature parameter matrix, and its No $\mathrm{j}$ column vectors of different feature parameters are $Z_{j}=$ $\left[z_{1 j}, z_{2 j}, \cdots, z_{n j}\right]^{T}$.

Furthermore, the mean value, root mean square, and kurtosis indices of roller vibration signals are expressed by $\bar{Y}, Y_{\mathrm{RMS}}$, and $K_{V}$, respectively. The frequency of barycenter, root mean square, and standard deviation of frequency related to the power spectrum of roller vibration signals are expressed by $F_{C}, \mathrm{RMSF}$, and RVF, respectively 


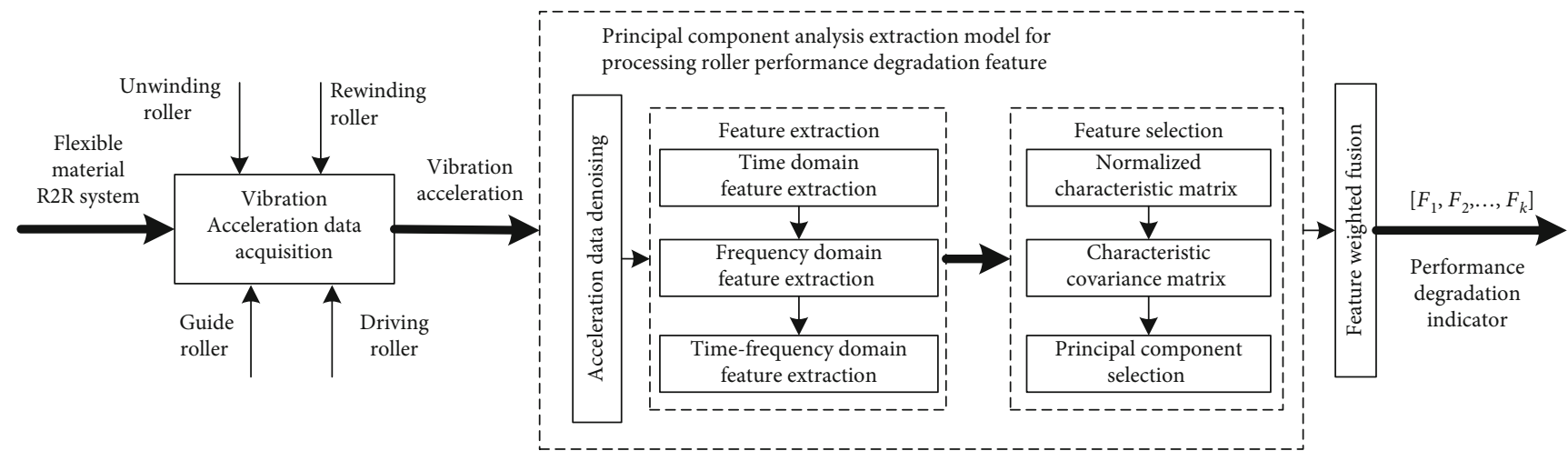

FIGURE 1: R2R processing roller shaft performance degradation feature extraction model framework.

[12]. The energy of intrinsic mode function components of vibration signals after EMD is represented by $E\left[\mathrm{IMF}_{1}\right]$, $E\left[\mathrm{IMF}_{2}\right], E\left[\mathrm{IMF}_{3}\right]$, and $E\left[\mathrm{IMF}_{4}\right]$ [13]. Take $n$ roller vibration signals and with $\bar{Y}, Y_{\mathrm{RMS}}, \mathrm{K}_{V}, F_{C}, \mathrm{RMSF}, \mathrm{RVF}, E[$
$\left.\mathrm{IMF}_{1}\right], E\left[\mathrm{IMF}_{2}\right], E\left[\mathrm{IMF}_{3}\right]$, and $E\left[\mathrm{IMF}_{4}\right]$ as parameters in equation (2), the original feature parameter matrix of the roller shafts' vibration signal $Z_{\text {org }}$ can be obtained as follows:

$$
Z_{\text {org }}=\left(\begin{array}{cccccccccc}
\bar{Y}_{1} & Y_{\mathrm{RMS} 1} & \mathrm{~K}_{V_{1}} & F_{C_{1}} & \mathrm{RMSF}_{1} & \mathrm{RVF}_{1} & E\left[\mathrm{IMF}_{1}\right]_{1} & E\left[\mathrm{IMF}_{2}\right]_{1} & E\left[\mathrm{IMF}_{3}\right]_{1} & E\left[\mathrm{IMF}_{4}\right]_{1} \\
\bar{Y}_{2} & Y_{\mathrm{RMS} 2} & \mathrm{~K}_{V_{2}} & F_{C_{2}} & \mathrm{RMSF}_{2} & \mathrm{RVF}_{2} & E\left[\mathrm{IMF}_{1}\right]_{2} & E\left[\mathrm{IMF}_{2}\right]_{2} & E\left[\mathrm{IMF}_{3}\right]_{2} & E\left[\mathrm{IMF}_{4}\right]_{2} \\
\vdots & \vdots & \vdots & \vdots & \vdots & \vdots & \vdots & \vdots & \vdots & \vdots \\
\bar{Y}_{n} & Y_{\mathrm{RMS} n} & \mathrm{~K}_{V_{n}} & F_{C_{n}} & \mathrm{RMSF}_{n} & \mathrm{RVF}_{n} & E\left[\mathrm{IMF}_{1}\right]_{n} & E\left[\mathrm{IMF}_{2}\right]_{n} & E\left[\mathrm{IMF}_{3}\right]_{n} & E\left[\mathrm{IMF}_{4}\right]_{n}
\end{array}\right) .
$$

In equation (3), the center of gravity frequency $F_{c}=$ $\int_{0}^{+\infty} f s(f) d f / \int_{0}^{+\infty} s(f) d f$, root mean square frequency RMSF $=\sqrt{\int_{0}^{+\infty} f^{2} s(f) d f / \int_{0}^{+\infty} s(f) d f}$, and frequency standard deviation $\operatorname{RVF}=\sqrt{\int_{0}^{+\infty}\left(f-F_{c}\right)^{2} s(f) d f / \int_{0}^{+\infty} s(f) d f}$, where $f$ is the vibration frequency and $s(f)$ is the power spectrum of the vibration signal.

3.2. Normalization of Feature Parameter Matrix. Since the parameters of feature parameter matrix $Z_{\text {org }}$ have different dimension and dimension units, in order to eliminate the influence of dimension between indexes on the accuracy of data analysis results, it is necessary to normalize feature parameter matrix so that each index is in the same order of magnitude [14].

For $n$ samples collected, $z_{k j}$ is an element of the feature parameter matrix $Z$; then, the mean value of feature parameters in column No j of feature parameter matrix $Z$ is obtained by $\bar{Z}_{j}=E\left(Z_{j}\right)=\sum_{k=1}^{n} z_{k j} / n$. For normalization of feature matrix $Z$, the variable $z_{k j}$ of No j column and No k row in feature matrix $Z$ is divided by the mean value $\bar{z}_{j}$ of the feature parameter in No $\mathrm{j}$ column in turn. That is, the normalized value $z_{k j}{ }^{*}$ of $z_{k j}$ is calculated by the following equation (4):

$$
z_{k j}^{*}=\frac{z_{k j}}{E\left(Z_{j}\right)}=\frac{z_{k j}}{\sum_{k=1}^{n} z_{k j} / n} \quad, j=1,2, \cdots, m .
$$

According to the above normalization method, the feature parameter matrix $Z$ represented by equation (2) is normalized to $Z^{(1)}$ as follows:

$$
Z^{(1)}=\left(\begin{array}{cccc}
z_{11}{ }^{*} & z_{12}{ }^{*} & \cdots & z_{1 m}{ }^{*} \\
z_{21}{ }^{*} & z_{22}{ }^{*} & \cdots & z_{2 m}{ }^{*} \\
\vdots & \vdots & \cdots & \vdots \\
z_{n 1}{ }^{*} & z_{n 2}{ }^{*} & \cdots & z_{n m}{ }^{*}
\end{array}\right)=\left(Z_{1}{ }^{*}, Z_{2}{ }^{*}, \cdots, Z_{m}{ }^{*}\right)
$$

Equations (4) and (5) are used to normalize the original feature parameter matrix $Z_{\text {org }}$ expressed by equation (3). Equation (4) is used to obtain the average value, root mean square, kurtosis index, center of gravity frequency, root mean square of frequency, standard deviation of 
TABLE 1: Feature vector table of roll vibration signal for flexible material R2R system.

\begin{tabular}{|c|c|c|}
\hline Num & Eigenvector type & Eigenvector expression \\
\hline 1 & Average value of vibration signal & $\bar{Y}^{*}=\left(\bar{Y}_{1}^{*}, \bar{Y}_{2}^{*}, \cdots, \bar{Y}_{n}^{*}\right)^{T}$ \\
\hline 2 & RMS of vibration signal & $Y_{\mathrm{RMS}^{*}}{ }^{*}=\left(Y_{\mathrm{RMS}_{1}}{ }^{*}, Y_{\mathrm{RMS}_{2}}{ }^{*}, \cdots, Y_{\mathrm{RMS}_{1}}{ }^{*}\right)^{T}$ \\
\hline 3 & Kurtosis of vibration signal & $\mathrm{K}_{V}^{*}=\left(\mathrm{K}_{V 1}{ }^{*}, \mathrm{~K}_{V 2}{ }^{*}, \cdots, \mathrm{K}_{V n}^{*}\right)^{T}$ \\
\hline 4 & $F_{\mathrm{C}}$ (frequency domain parameters) & $F_{C}{ }^{*}=\left(F_{C_{1}}{ }^{*}, F_{C_{2}}{ }^{*}, \cdots, F_{C_{n}}{ }^{*}\right)^{T}$ \\
\hline 5 & RMSF (frequency domain parameters) & $\operatorname{RMSF}^{*}=\left(\operatorname{RMSF}_{C_{1}}{ }^{*}, \operatorname{RMSF}_{C_{2}}{ }^{*}, \cdots, \operatorname{RMSF}_{C_{n}}{ }^{*}\right)^{T}$ \\
\hline 6 & RVF (frequency domain parameters) & $\mathrm{RVF}^{*}=\left(\mathrm{RVF}_{1}{ }^{*}, \mathrm{RVF}_{2}{ }^{*}, \cdots, \mathrm{RVF}_{n}{ }^{*}\right)^{T}$ \\
\hline 7 & The energy of $\mathrm{IMF}_{1}$ & $E\left[\mathrm{IMF}_{1}\right]^{*}=\left(E\left[\mathrm{IMF}_{1}\right]_{1}^{*}, E\left[\mathrm{IMF}_{1}\right]_{2}^{*}, \cdots, E\left[\mathrm{IMF}_{1}\right]_{n}^{*}\right)^{T}$ \\
\hline 8 & The energy of $\mathrm{IMF}_{2}$ & $E\left[\mathrm{IMF}_{2}\right]^{*}=\left(E\left[\mathrm{IMF}_{2}\right]_{1}^{*}, E\left[\mathrm{IMF}_{2}\right]_{2}^{*}, \cdots, E\left[\mathrm{IMF}_{2}\right]_{n}^{*}\right)^{T}$ \\
\hline 9 & The energy of $\mathrm{IMF}_{3}$ & $E\left[\mathrm{IMF}_{3}\right]^{*}=\left(E\left[\mathrm{IMF}_{3}\right]_{1}^{*}, E\left[\mathrm{IMF}_{3}\right]_{2}^{*}, \cdots, E\left[\mathrm{IMF}_{3}\right]_{n}^{*}\right)^{T}$ \\
\hline 10 & The energy of $\mathrm{IMF}_{4}$ & $E\left[\mathrm{IMF}_{4}\right]^{*}=\left(E\left[\mathrm{IMF}_{4}\right]_{1}{ }^{*}, E\left[\mathrm{IMF}_{4}\right]_{2}{ }^{*}, \cdots, E\left[\mathrm{IMF}_{4}\right]_{n}{ }^{*}\right)^{T}$ \\
\hline
\end{tabular}

frequency, and the energy of intrinsic mode function components of roller vibration signal processed by flexible material R2R system: $\bar{Y}_{j}{ }^{*}, Y_{\mathrm{RMS}_{j}}{ }^{*}, \mathrm{~K}_{V_{j}}{ }^{*}, F_{C_{j}}{ }^{*}, \mathrm{RMSF}_{j}{ }^{*}$, and $\mathrm{RVF}_{j}{ }^{*}$ and $E\left[\mathrm{IMF}_{1}\right]_{j}{ }^{*}, E\left[\mathrm{IMF}_{2}\right]_{j}{ }^{*}, E\left[\mathrm{IMF}_{3}\right]_{j}{ }^{*}$, and $E$
$\left[\mathrm{IMF}_{4}\right]_{j}{ }^{*}$. Equation (5) is used to obtain the normalized matrix $Z_{\text {org }}{ }^{*}$ of the original feature parameter matrix $Z_{\text {org }}$ of the vibration signal of $\mathrm{R} 2 \mathrm{R}$ processing roll for flexible material is expressed as equation (6).

$$
Z_{\mathrm{org}}{ }^{*}=\left(\begin{array}{cccccccccc}
\bar{Y}_{1}^{*} & Y_{\mathrm{RMS}_{1}}{ }^{*} & K_{V_{1}}{ }^{*} & F_{C_{1}}{ }^{*} & \mathrm{RMSF}_{1}^{*} & \mathrm{RVF}_{1}^{*} & E\left[\mathrm{IMF}_{1}\right]_{1}^{*} & E\left[\mathrm{IMF}_{2}\right]_{1}^{*} & E\left[\mathrm{IMF}_{3}\right]_{1}^{*} & E\left[\mathrm{IMF}_{4}\right]_{1}^{*} \\
\bar{Y}_{2}^{*} & Y_{\mathrm{RMS}_{2}}{ }^{*} & K_{V_{2}}{ }^{*} & F_{C_{2}}{ }^{*} & \mathrm{RMSF}_{2}^{*} & \mathrm{RVF}_{2}^{*} & E\left[\mathrm{IMF}_{1}\right]_{2}^{*} & E\left[\mathrm{IMF}_{2}\right]_{2}^{*} & E\left[\mathrm{IMF}_{3}\right]_{2}^{*} & E\left[\mathrm{IMF}_{4}\right]_{2}^{*} \\
\vdots & \vdots & \vdots & \vdots & \vdots & \vdots & \vdots & \vdots & \vdots & \vdots \\
\bar{Y}_{n}^{*} & Y_{\mathrm{RMS}_{n}}{ }^{*} & K_{V_{n}}{ }^{*} & F_{C_{n}}{ }^{*} & \mathrm{RMSF}_{n}^{*} & \mathrm{RVF}_{n}^{*} & E\left[\mathrm{IMF}_{1}\right]_{n}^{*} & E\left[\mathrm{IMF}_{2}\right]_{n}^{*} & E\left[\mathrm{IMF}_{3}\right]_{n}^{*} & E\left[\mathrm{IMF}_{4}\right]_{n}^{*}
\end{array}\right) .
$$

Based on equation (6), it can be seen in Table 1 that each column vector in the feature parameter matrix $Z_{\text {org }}{ }^{*}$ represents one of the eigenvectors of the vibration signal of $R 2 R$ processing roll for flexible material.

3.3. Construction of Covariance Matrix of Feature Parameters and Screening of Feature Values. The feature parameter matrix of vibration signal of R2R processing roll for flexible material has been deduced above. However, in order to obtain the principal component variable of the feature parameters of the vibration signal of the processing roller, it is necessary to construct the feature quantity covariance matrix of $Z_{\mathrm{org}}{ }^{*}$ and screen the feature values.

According to the definition of the covariance matrix, the correlation covariance matrix between two parameters in feature parameter matrix $Z_{\mathrm{org}}{ }^{*}$ can be expressed as $C_{Z}$ of equation (7):

$$
C_{Z}=\left[\begin{array}{ccc}
\operatorname{cov}\left(\bar{Y}^{*}, \bar{Y}^{*}\right) & \cdots & \operatorname{cov}\left(\bar{Y}^{*}, E\left[\mathrm{IMF}_{4}\right]^{*}\right) \\
\vdots & \ddots & \vdots \\
\operatorname{cov}\left(E\left[\mathrm{IMF}_{4}\right]^{*}, \bar{Y}^{*}\right) & \cdots & \operatorname{cov}\left(E\left[\mathrm{IMF}_{4}\right]^{*}, E\left[\mathrm{IMF}_{4}\right]^{*}\right)
\end{array}\right] .
$$

Because the covariance matrix is a symmetric matrix and the diagonal line of the matrix is the variance of each dimension, it not only reflects the correlation measure between all feature dimensions but also reflects the degree of data redundancy. The larger the value on the diagonal line of the matrix, the variables are more important. Conversely, the smaller the value, the smaller the corresponding variable is the secondary variable of the noise signal [15-17].

Next, the covariance matrix $C_{Z}$ is transformed into a diagonal matrix by Jacobi iteration method [18], and then, all eigenvalues are solved. Assuming that the eigenvalue of the covariance matrix is $\lambda_{i}(i=1,2, \cdots, m)$, it is regarded as a diagonal matrix composed of principal diagonal elements, and $\Lambda$ is used to represent the orthogonal matrix composed of the eigenvectors corresponding to eigenvalues. According to the property of symmetric matrix $R^{T} C_{Z} R=\Lambda$, thus the problem of finding eigenvalues of covariance matrix $C_{Z}$ is transformed into solving orthogonal matrix $R$ [19]. Furthermore, according to the iteration principle of Jacobi's method, the orthogonal transformation of covariance matrix can be realized by constructing a series of planar rotating orthogonal matrices so that the proportion of diagonal elements of the covariance 

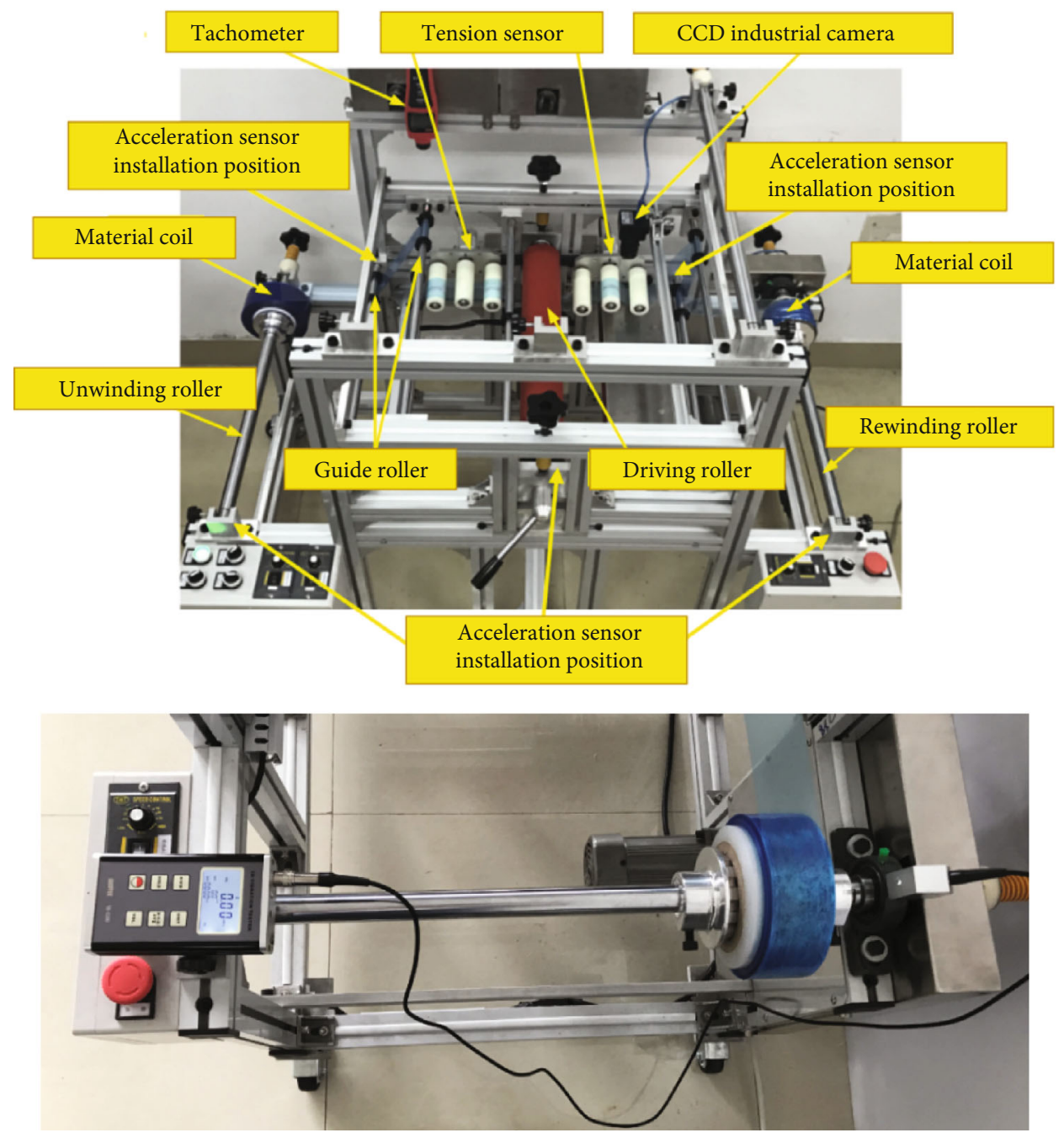

FIGURE 2: Flexible material roll-to-roll processing equipment.

matrix $C_{Z}$ increases step by step, while the nondiagonal elements become smaller. When the nondiagonal elements approach zero, the principal diagonal elements can be approximated as matrices. Eigenvalues of $C_{Z}$ and expressions of matrix $\Lambda$ obtained from diagonalization of covariance matrix $C_{Z}$ are as follows:

$$
\Lambda=\left[\begin{array}{cccc}
\lambda_{1} & 0 & \cdots & 0 \\
0 & \lambda_{2} & \cdots & 0 \\
\vdots & \vdots & \ddots & 0 \\
0 & 0 & \cdots & \lambda_{m}
\end{array}\right]
$$

In order to solve the diagonal matrix $\Lambda$ of equation (8), it is necessary to construct a rotating orthogonal matrix to transform covariance matrix $C_{Z}$ orthogonally. The rotation angle of the covariance matrix [20] is $\theta$, and the subscripts of the absolute maximum element of covariance matrix $C_{Z}$ on nonprincipal diagonal line are expressed by $p$ and $q$, respectively. The rotating orthogonal matrix $R_{p q}(\theta)$ is constructed:

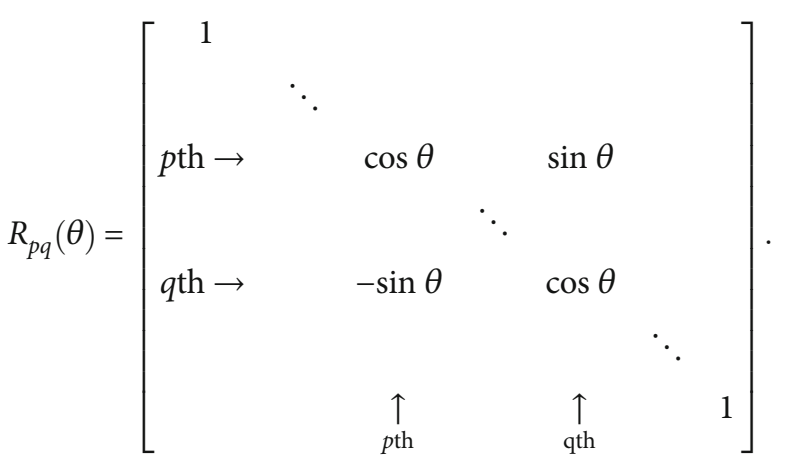

Further, if the initial value of the covariance matrix is $C_{Z}{ }^{0}$ and the first rotating orthogonal matrix $R_{p q}^{(1)}(\theta)$ is 


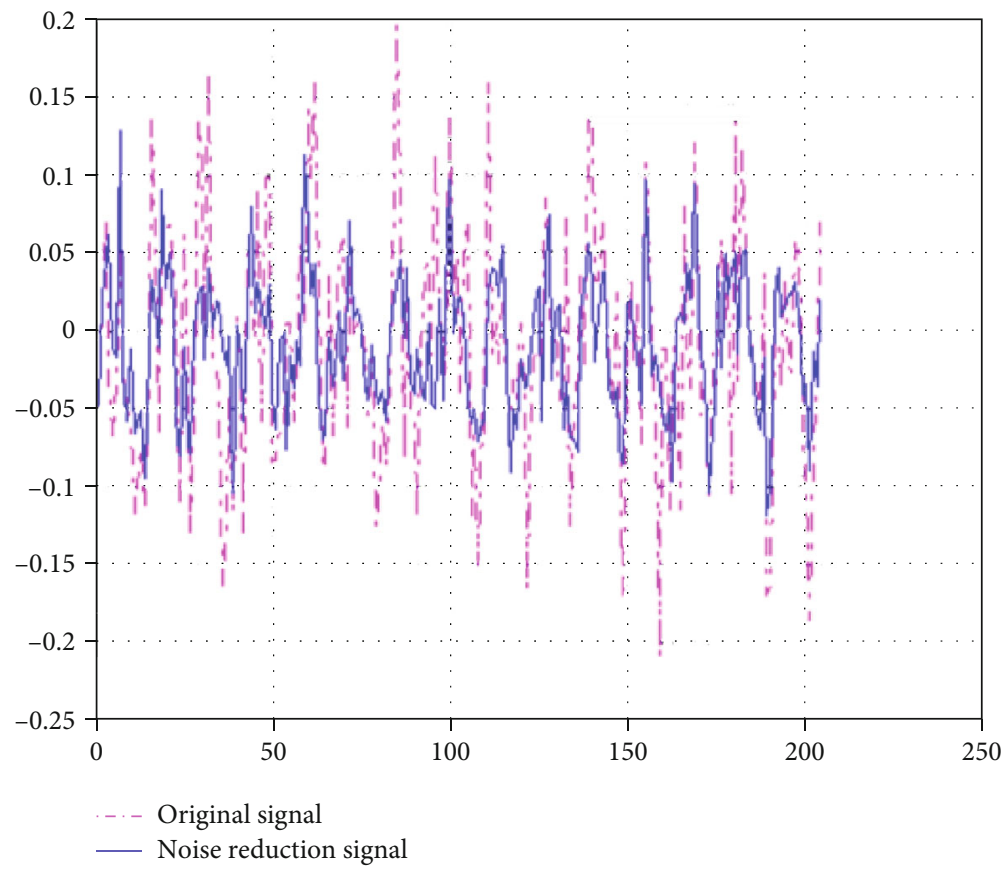

Figure 3: Moving average filtering effect diagram.

constructed, the covariance matrix $C_{Z}$ is transformed into $C_{Z}{ }^{(1)}$ by the first orthogonal transformation.

$$
C_{Z}^{(1)}=R_{p q}^{(1)_{T}}(\theta) C_{Z}^{(0)} R_{p q}^{(1)}(\theta) .
$$

The element values of No $j$ column and No i row of matrix $C_{Z}{ }^{(1)}$ and $C_{Z}{ }^{0}$ are represented by $c^{(1)}{ }_{i j}$ and $c^{(0)}{ }_{i j}$, respectively. Equation (9) is substituted in equation (10) and expanded to obtain as equation (11)

$$
\left\{\begin{array}{l}
c^{(1)}{ }_{i p}=c^{(1)}{ }_{p i}=c^{(0)}{ }_{p i} \cos \theta-c^{(0)}{ }_{q i} \sin \theta, i \neq p, q, \\
c^{(1)}{ }_{i q}=c^{(1)}{ }_{q i}=c^{(0)}{ }_{p i} \sin \theta+c^{(0)}{ }_{q i} \cos \theta, i \neq p, q, \\
c^{(1)}{ }_{p p}=c^{(0)}{ }_{p p} \cos ^{2} \theta+c^{(0)}{ }_{q q} \sin ^{2} \theta-c^{(0)}{ }_{p q} \sin 2 \theta, \\
c^{(1)}{ }_{q q}=c^{(0)}{ }_{p p} \sin ^{2} \theta+c^{(0)}{ }_{q q} \cos ^{2} \theta+c^{(0)}{ }_{p q} \sin 2 \theta, \\
c^{(1)}{ }_{p q}=c^{(1)}{ }_{q p}=c^{(0)}{ }_{p q} \cos 2 \theta+\frac{c^{(0)}{ }_{p p}-c^{(0)}{ }_{q q}}{2} \sin 2 \theta .
\end{array}\right.
$$

Furthermore, to reduce nondiagonal element component, set $c^{(1)}{ }_{p q}=c^{(1)}{ }_{q p}=0$, introduce the variables $s$ and $t$, and assume $s=c^{(0)}{ }_{q q}-c^{(0)}{ }_{p p} / 2 c^{(0)}{ }_{p q}, t=\tan \theta$. According to trigonometric function $t=\left\{\begin{array}{l}t^{2}+2 t s-1=0, s \neq 0 \\ 1, s=0\end{array}, g\right.$ is used to denote $\cos \theta=1 /\left(\sqrt{1+t^{2}}\right)=g$, and $h$ is used to represent $\sin \theta=t /\left(\sqrt{1+t^{2}}\right)=h$; then, equation (11) is simplified as [21] equation (12). Equation (12) is the matrix after the first orthogonal transformation.

$$
\left\{\begin{array}{l}
c^{(1)}{ }_{i p}=c^{(1)}{ }_{p i}=g c^{(0)}{ }_{p i}-h c^{(0)}{ }_{q i}, i \neq p, q, \\
c^{(1)}{ }_{i q}=c^{(1)}{ }_{q i}=h c^{(0)}{ }_{p i}+g c^{(0)}{ }_{q i}, i \neq p, q, \\
c^{(1)}{ }_{p p}=c^{(0)}{ }_{p p}-t c^{(0)}{ }_{p q}, \\
c^{(1)}{ }_{q q}=c^{(0)}{ }_{q q}+t c^{(0)}{ }_{p q}, \\
c^{(1)}{ }_{p q}=c^{(1)}{ }_{q p}=0 .
\end{array}\right.
$$

Further, iteratively calculates $C_{Z}^{(1)}$, according to $c_{p q}^{(k-1)}\left|=\max _{i \neq j}\right| c^{(k-1)}{ }_{i j} \mid$, the subscript of the largest element of absolute value on nonmain diagonal of the rotation matrix $R_{p q}^{(k-1)}(\theta)$ is selected as the $p$ and $q$ values of rotation matrix $R_{p q}^{(k)}(\theta)$. The covariance matrix $C_{Z}$ is obtained after $k$ times of orthogonal transformations equation (13).

$$
\begin{aligned}
C_{Z}{ }^{(k)} & =R_{p q}^{(k)_{T}}(\theta) C_{Z}{ }^{(k-1)} R_{p q}^{(k)}(\theta) \\
& =R_{p q}^{(k)_{T}} R_{p q}^{(k-1)_{T}} \cdots R_{p q}^{(1)_{T}} C_{Z}{ }^{(0)} R_{p q}^{(1)}(\theta) \cdots R_{p q}^{(k-1)}(\theta) R_{p q}^{(k)}(\theta) .
\end{aligned}
$$




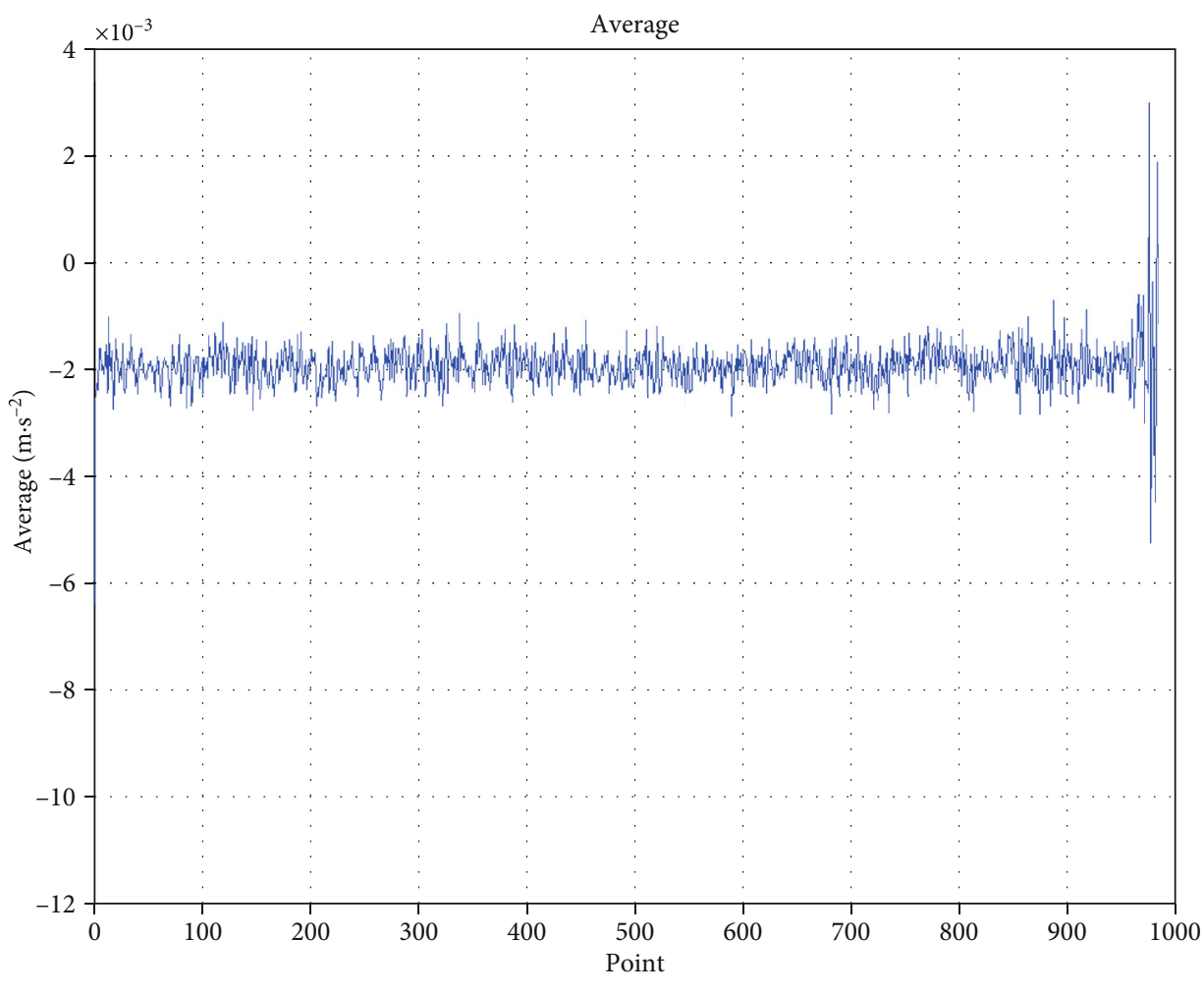

- Average

(a) Average value

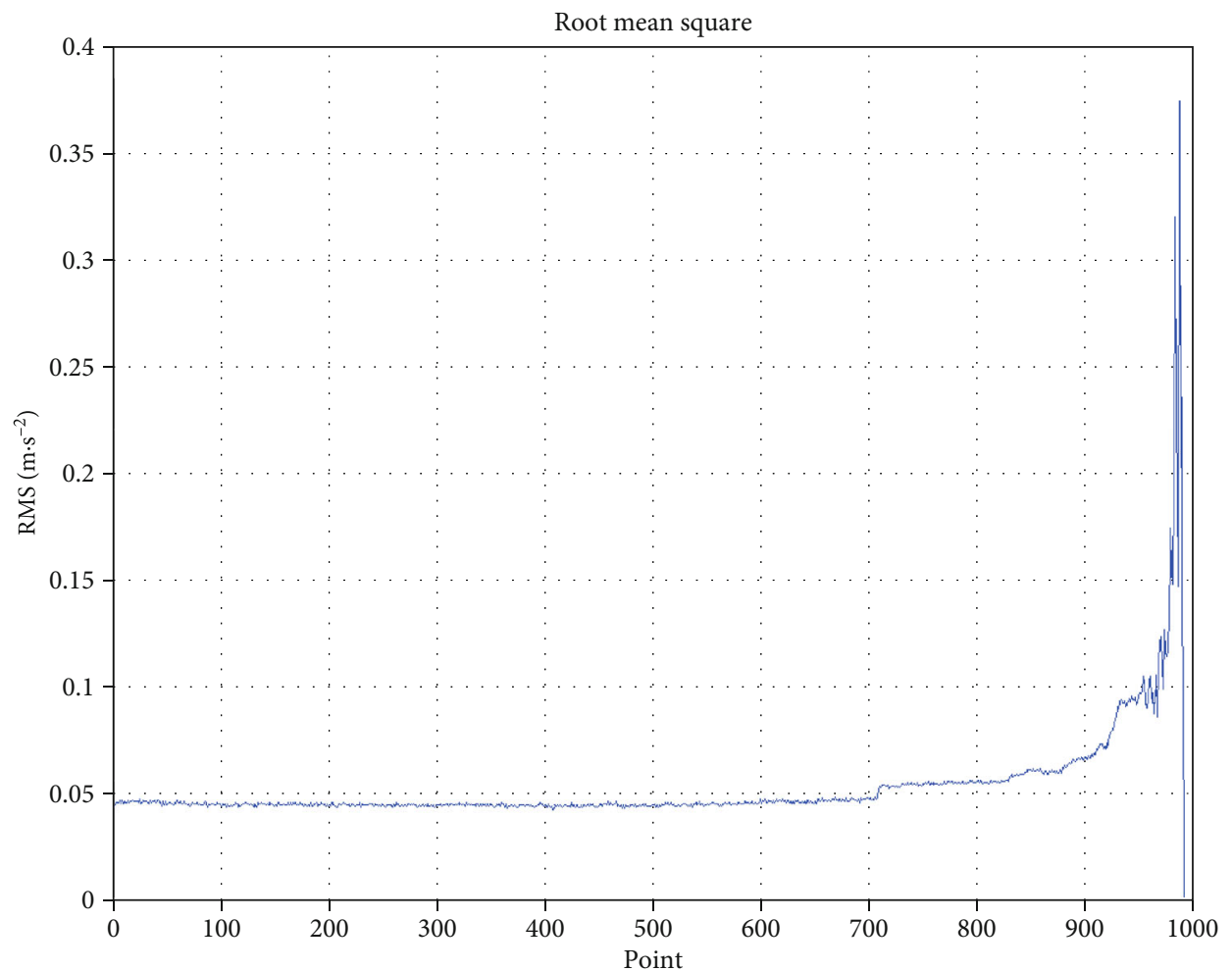

— RMS

(b) Root mean square

FIgURe 4: Continued. 


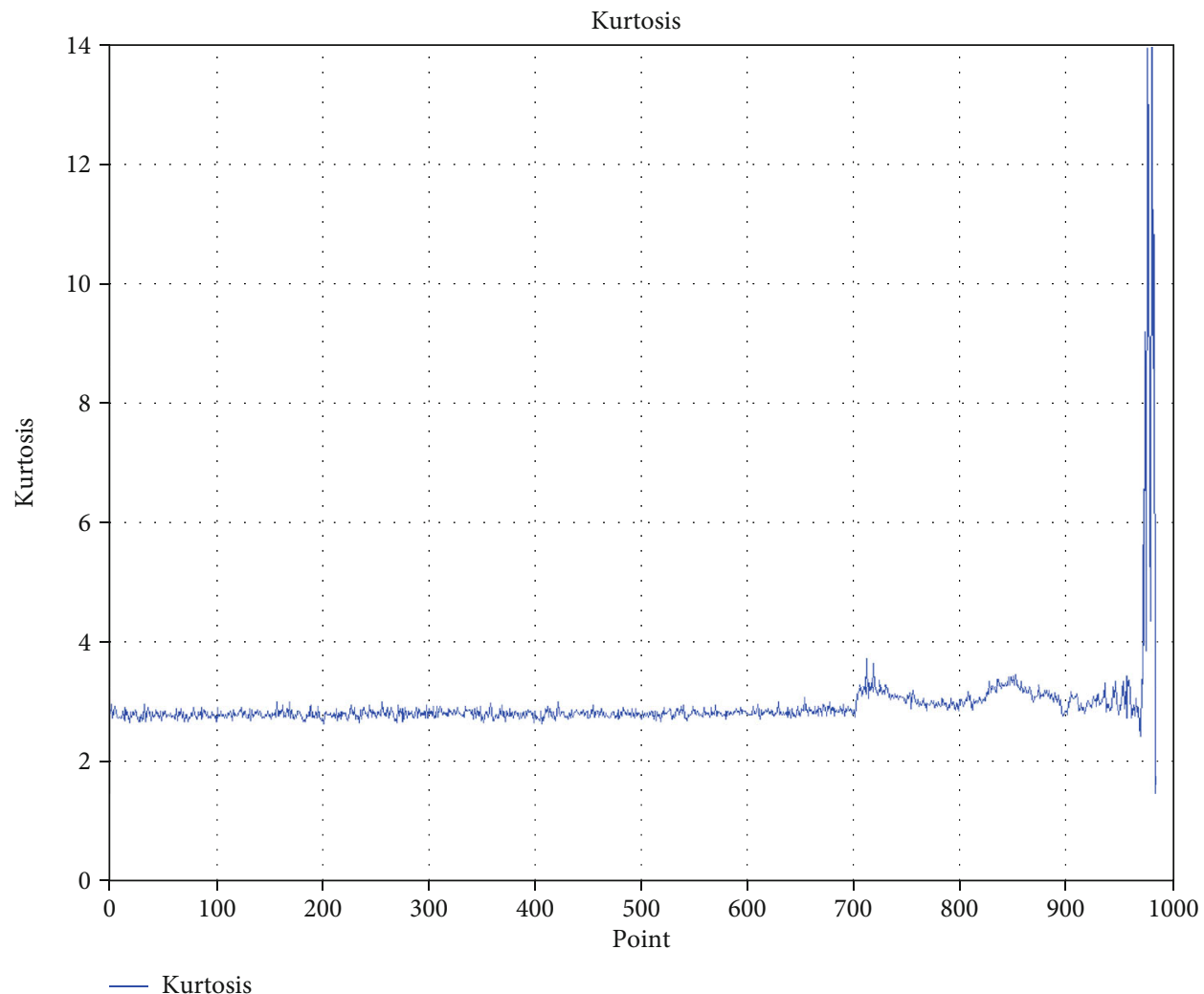

(c) Kurtosis value

Figure 4: The calculation results of mean value, root mean square, and kurtosis index.

If the form of the covariance matrix $C_{Z}{ }^{(k)}$ is consistent with the form of the symmetric matrix shown in equation (8), terminate iterating. $C_{Z}{ }^{(k)}$ is obtained as equation (14).

$$
C_{Z}{ }^{(k)}=\left[\begin{array}{cccc}
\lambda_{1} & 0 & \cdots & 0 \\
0 & \lambda_{2} & \cdots & 0 \\
\vdots & \vdots & \ddots & 0 \\
0 & 0 & \cdots & \lambda_{k}
\end{array}\right] .
$$

According to the properties of symmetric matrices, we further derive from equation (14) and have equation (15):

$$
C_{Z}^{(k)}=\Lambda=R^{T} C_{Z} R
$$

In equation (15), the relationship between the orthogonal matrix $R$ and rotating orthogonal matrix $R_{p q}^{(k)}(\theta)$ of equation (13) is $R=R_{p q}^{(1)}(\theta) \cdots R_{p q}^{(k)}(\theta) \cdots R_{p q}^{(n)}($ $\theta$ ), and the principal diagonal element of $C_{Z}{ }^{(k)}$ is all the eigenvalues of the covariance matrix $C_{Z}$.

In order to better screen eigenvalues of covariance matrix $C_{Z}$, it is necessary to calculate the cumulative contribution rate of eigenvalues. Let $\lambda_{i}$ denote eigenvalue of covariance matrix $C_{Z}$, the cumulative sum of the first eigenvalues is $\sum_{i=1}^{l} \lambda_{i}$, and cumulative sum of all eigenvalues is $\sum_{i=1}^{m} \lambda_{i}, l$ $<m$. Then, the principal component value (or variance ratio) of single eigenvalue is calculated by $\lambda_{i} / \sum_{i=1}^{m} \lambda_{i}$. The cumulative contribution rate $\eta$ of eigenvalues is calculated by the following equation (16). The constant $\varepsilon=0.85$ is introduced as the screening condition in this paper, and the principal components are retained only when $\eta \geq \varepsilon$ is satisfied, which is used for subsequent weighted fusion calculation of eigenvectors [4].

$$
\eta=\frac{\left(\sum_{i=1}^{l} \lambda_{i}\right)}{\left(\sum_{i=1}^{m} \lambda_{i}\right)}, \quad l<m .
$$

3.4. Derivation of Principal Component Selection Algorithms for Feature Matrix. The normalization of the original feature parameter matrix and screening method of covariance matrix eigenvalue of vibration signal are discussed before. The algorithm of selecting principal components of the feature matrix of the vibration signal of processing roll is deduced below.

The eigenvector matrix consisting of eigenvectors corresponding to selected eigenvalues is represented by ${ }_{U}$. The eigenvector matrix is expressed as follows when $U_{i}=\left[u_{1 i}, u_{2 i}, \cdots, u_{r i}\right]^{T}$ is used to represent the eigenvectors of No i column ( $r$ is used to represent the number of eigenvalues 


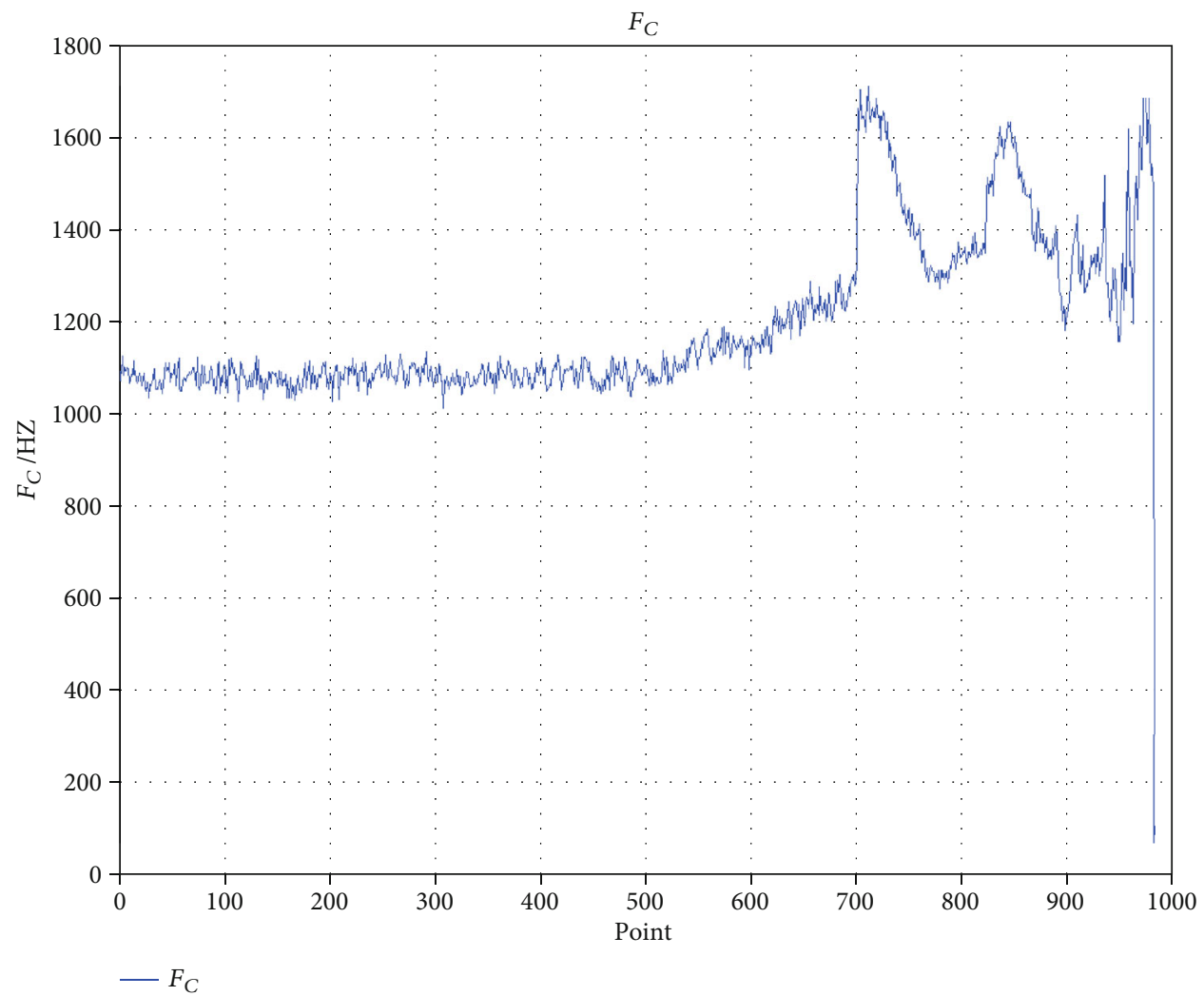

(a) Centre of gravity frequency

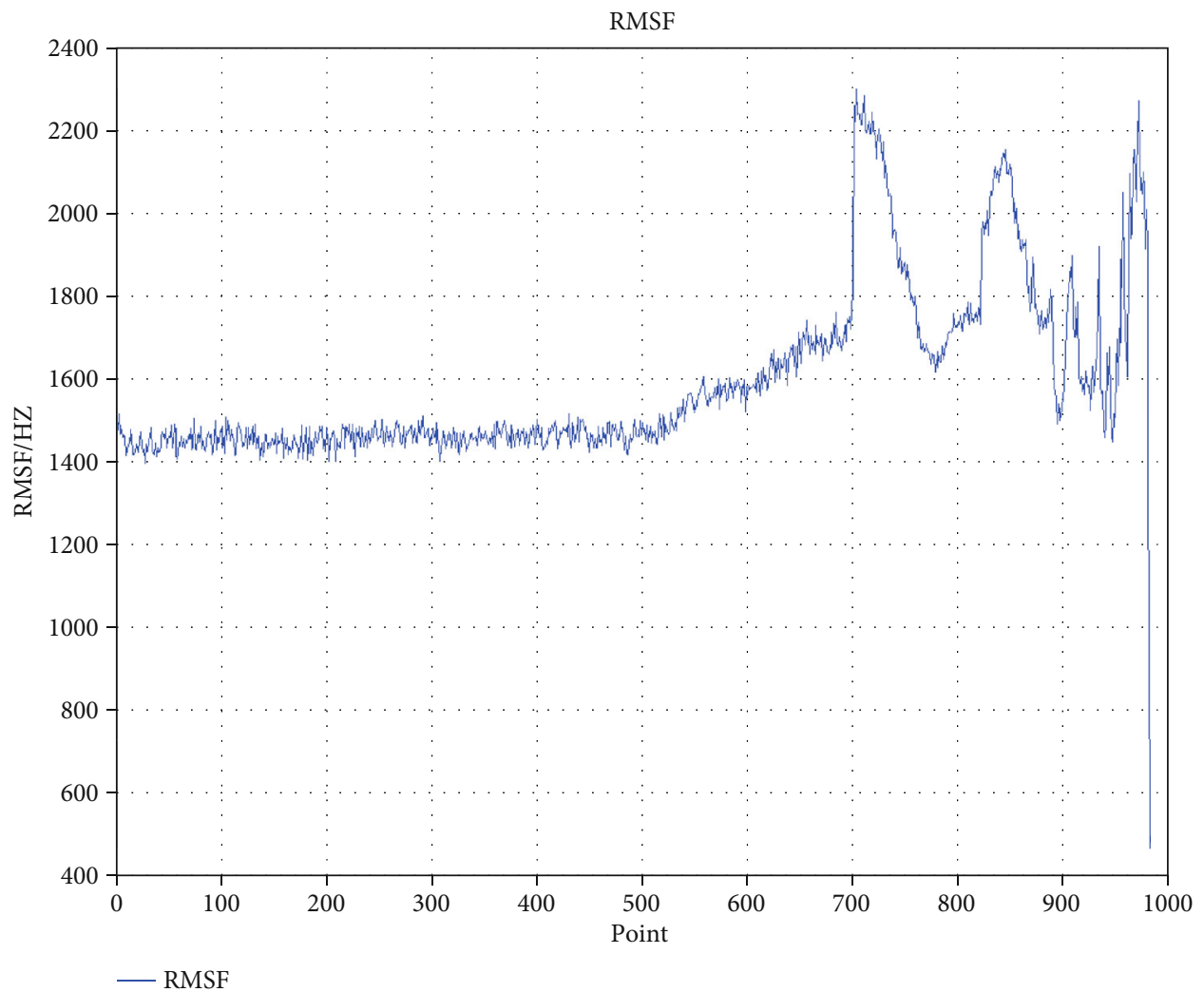

(b) RMS of frequency

Figure 5: Continued. 


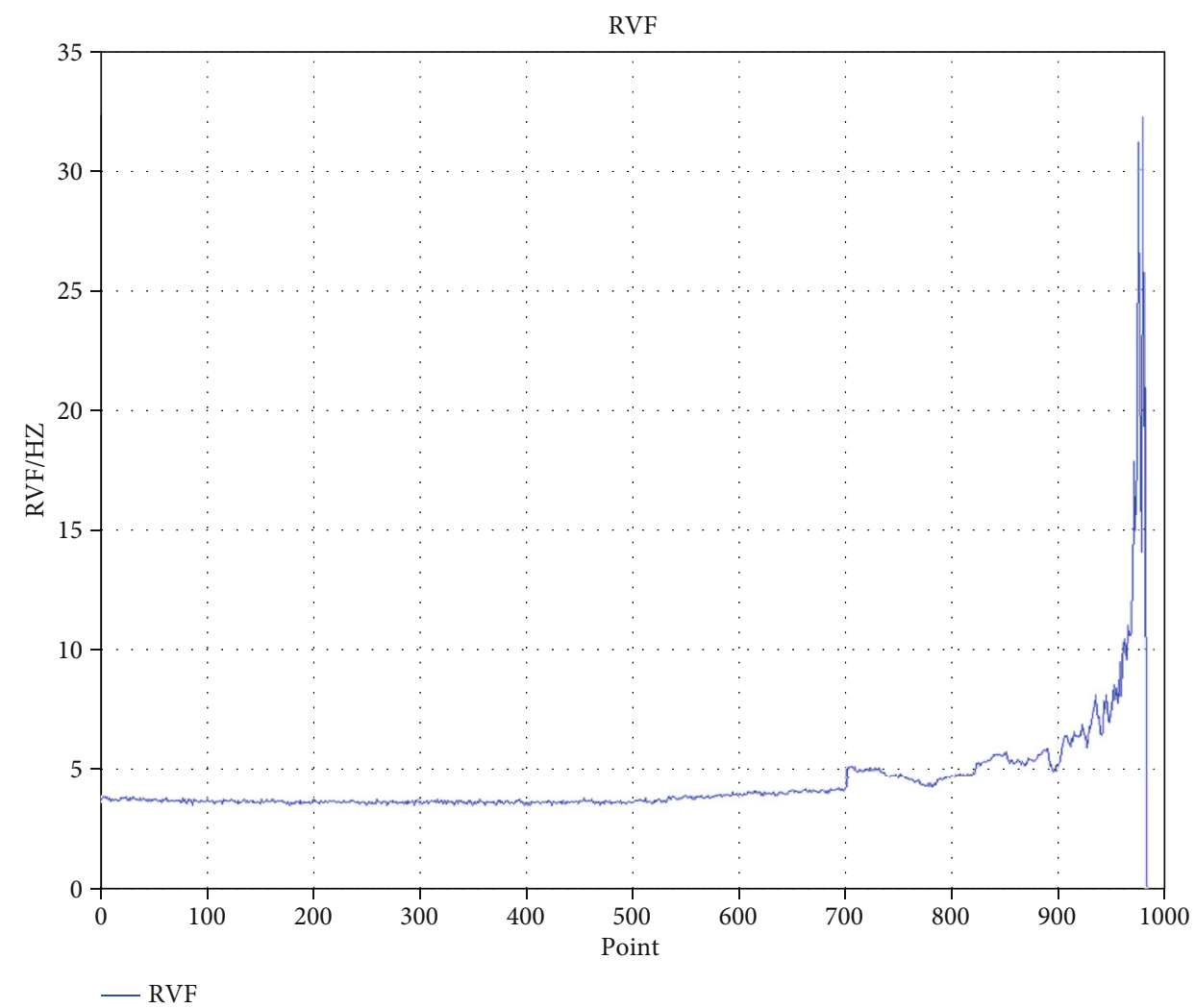

(c) Frequency standard deviation

FigURE 5: Calculations of center of gravity frequency, frequency root mean square, and frequency standard deviation.

contained in each column of eigenvectors) shown as equation (17).

$$
U=\left(U_{1}, U_{2}, \cdots, U_{l}\right)=\left(\begin{array}{cccc}
u_{11} & u_{12} & \cdots & u_{1 l} \\
u_{21} & u_{22} & \cdots & u_{2 l} \\
\vdots & \vdots & \cdots & \vdots \\
u_{r 1} & u_{r 2} & \cdots & u_{r l}
\end{array}\right) .
$$

Then, the normalized matrix $Z_{\mathrm{org}}{ }^{*}$ of original feature parameters shown in equation (6) and the eigenvector matrix
$U$ are calculated by linear weighting fusion, and feature principal component matrix $F$ of the vibration signal is obtained as equation (18).

Thus, the first principal component, the second principal component,..., the No $l$ principal component of the vibration signal features $F_{1}, F_{2}, \cdots, F_{l}$ can be obtained, which is calculated as equation (19).

To this point, the principal component extraction method of vibration signal features of $\mathrm{R} 2 \mathrm{R}$ processing roll for flexible material has been deduced. The validity of the above aspects is verified by experiments.

$$
\begin{aligned}
& F=Z_{\text {org }}{ }^{*} \bullet U \Rightarrow
\end{aligned}
$$

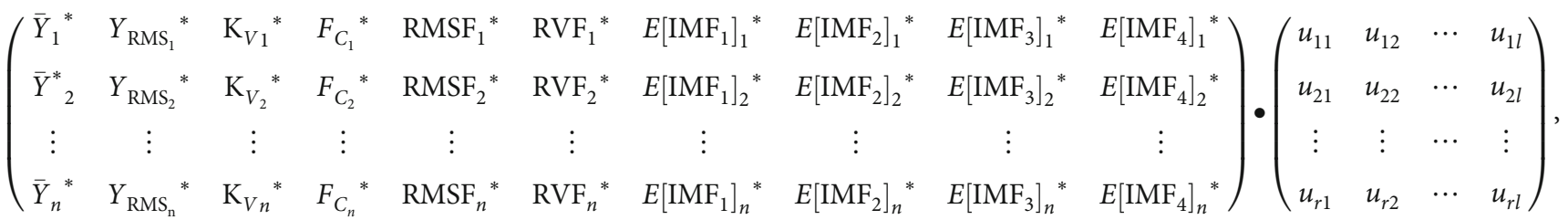

$$
\begin{aligned}
& \left\{\begin{array}{l}
F_{1}=u_{11} \bar{Y}^{*}{ }_{1}+u_{21} Y_{\mathrm{RMS}_{1}}{ }^{*}+\cdots+u_{r 1} E\left[\mathrm{IMF}_{4}\right]_{1}{ }^{*}, \\
F_{2}=u_{12} \bar{Y}^{*}{ }_{2}+u_{22} Y_{\mathrm{RMS}_{2}}{ }^{*}+\cdots+u_{r 2} E\left[\mathrm{IMF}_{4}\right]_{2}{ }^{*}, \\
\vdots \\
F_{l}=u_{1 l} \bar{Y}_{n}^{*}+u_{2 l} Y_{\mathrm{RMS}^{*}}{ }_{n}+\cdots+u_{r l} E\left[\mathrm{IMF}_{4}\right]_{n}{ }^{*} .
\end{array}\right.
\end{aligned}
$$




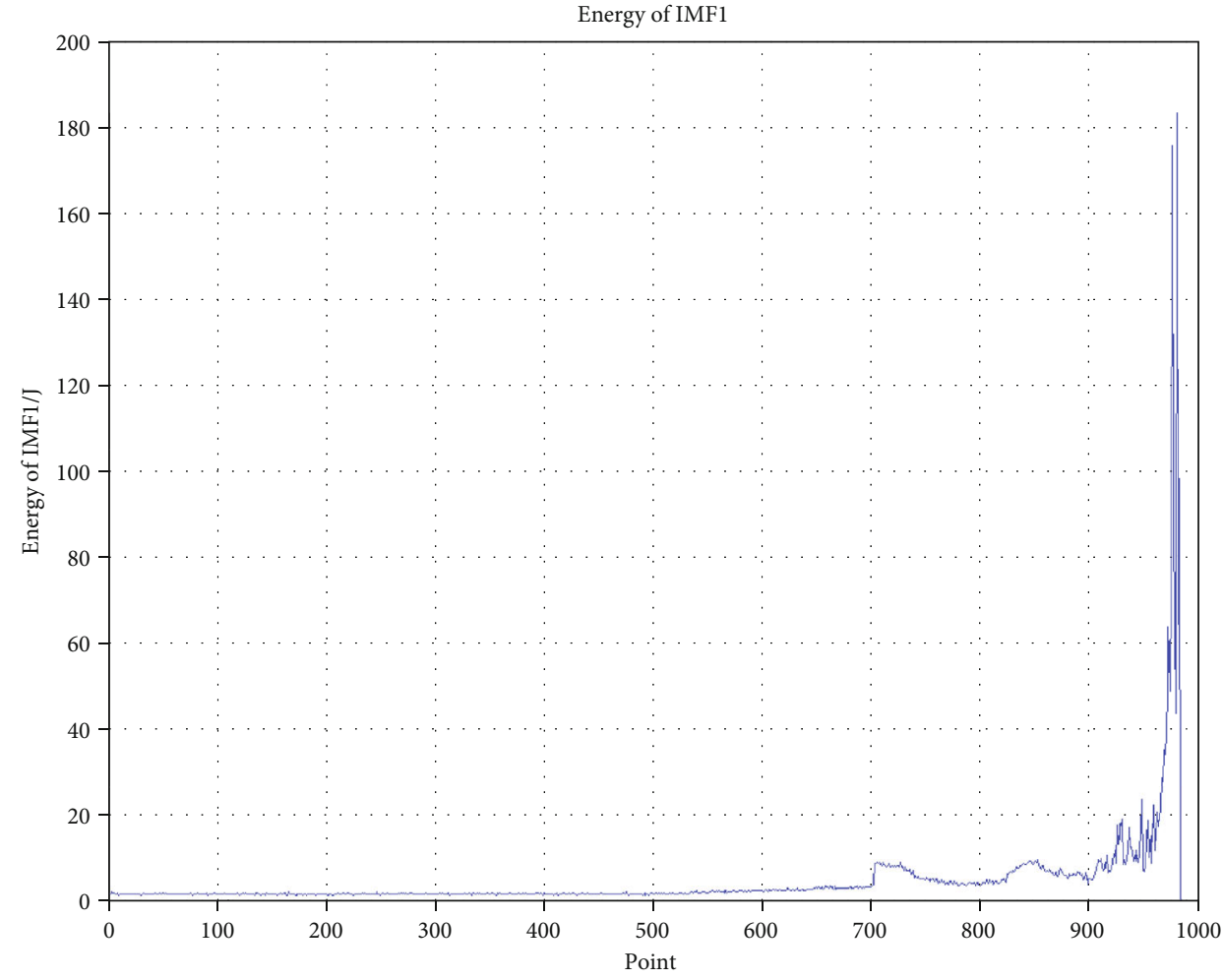

— Energy of IMF1

(a) Energy of IMF1

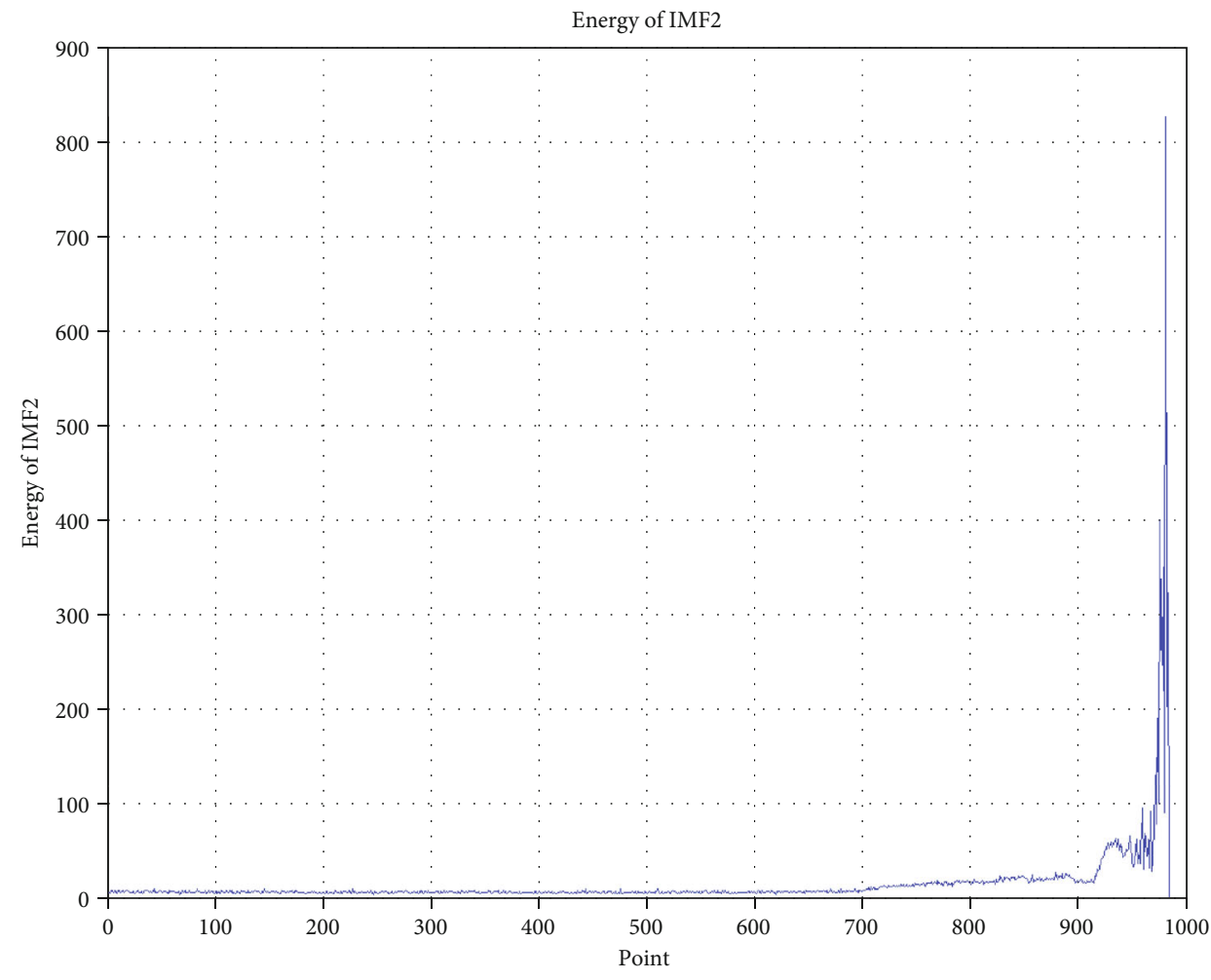

_ Energy of IMF2

(b) Energy of IMF2

FIgUre 6: Continued. 


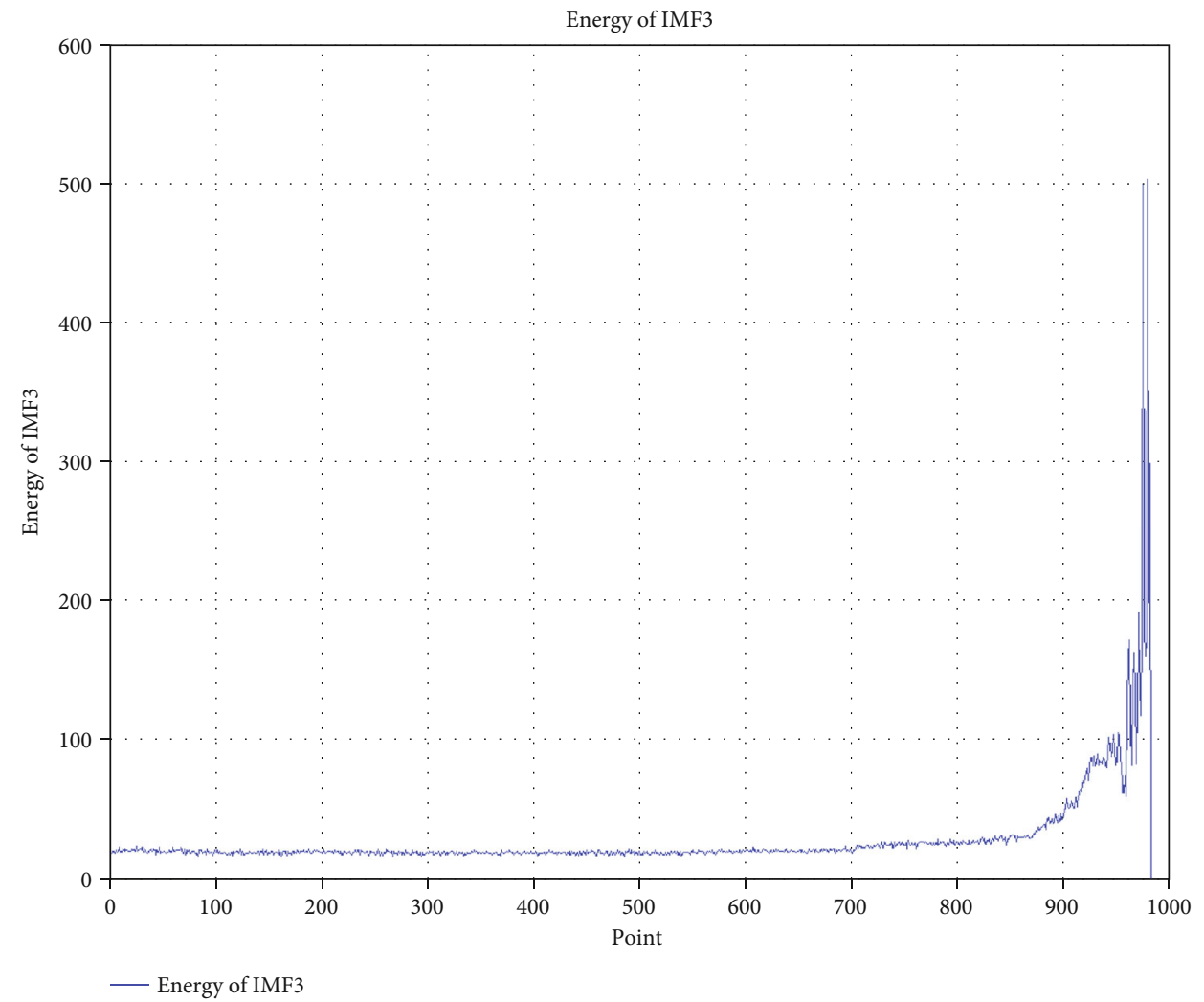

(c) Energy of IMF3

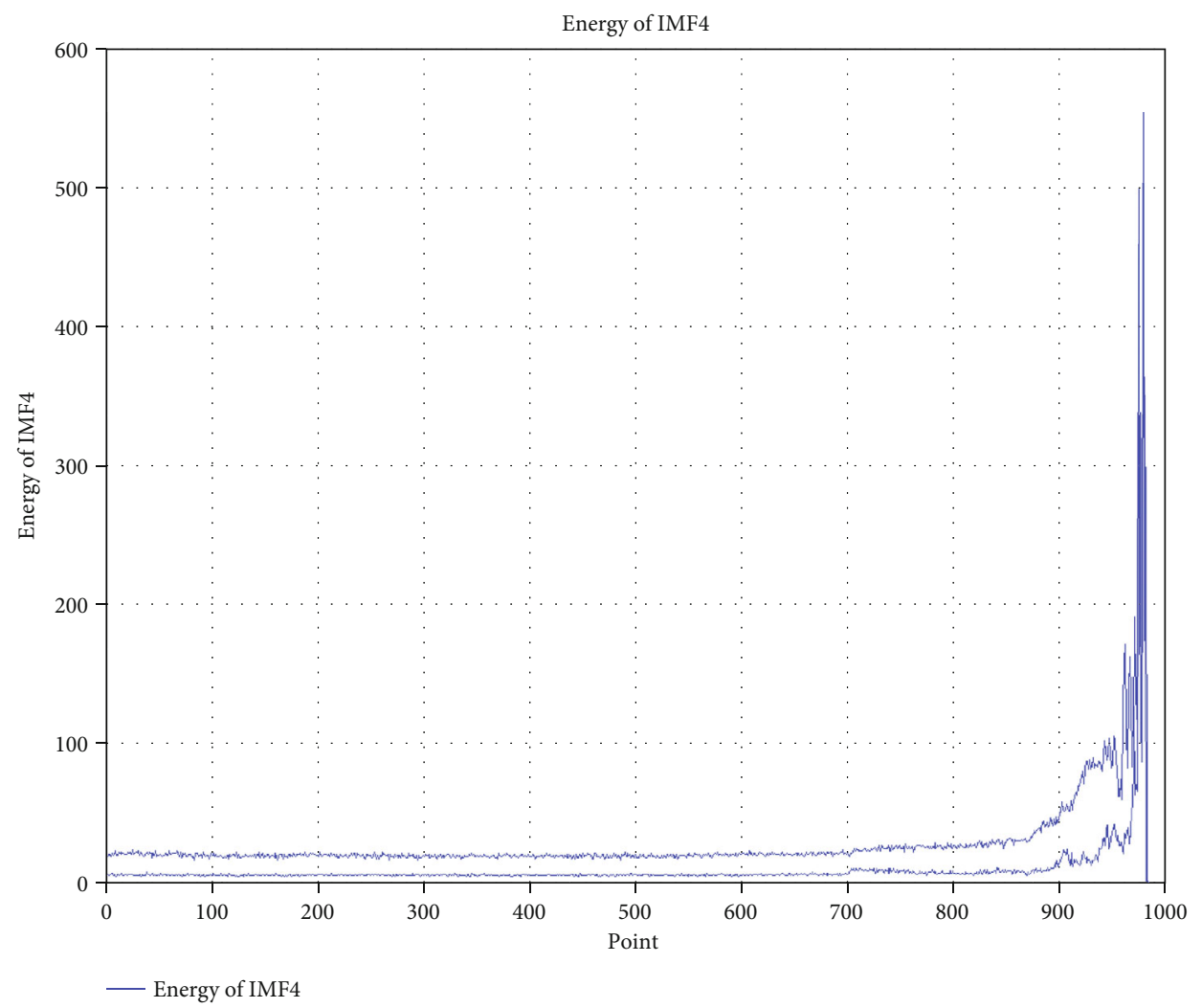

(d) Energy of IMF4

FIGURE 6: Calculation results of intrinsic mode function components. 
TABLE 2: Original feature parameter matrix for flexible material R2R system roller vibration signal.

\begin{tabular}{|c|c|c|c|c|c|c|c|c|c|c|}
\hline Num & $\bar{Y}$ & $Y_{\mathrm{RMS}}$ & $\mathrm{K}_{V}$ & $F_{C}$ & RMSF & RVF & $E\left[\mathrm{IMF}_{1}\right]$ & $E\left[\mathrm{IMF}_{2}\right]$ & $E\left[\mathrm{IMF}_{3}\right]$ & $E\left[\mathrm{IMF}_{4}\right]$ \\
\hline 1 & -0.0101 & 0.0448 & 2.7899 & 1050.3 & 1450.9 & 3.6648 & 1.5456 & 7.65 & 18.466 & 4.3143 \\
\hline 2 & -0.0025 & 0.0438 & 2.9485 & 1090.8 & 1500.4 & 3.7297 & 1.464 & 6.3859 & 18.301 & 5.1165 \\
\hline 3 & -0.0024 & 0.0450 & 2.7206 & 1086.4 & 1475.1 & 3.7481 & 1.6174 & 6.5097 & 19.865 & 4.8536 \\
\hline 4 & -0.0022 & 0.0456 & 2.8154 & 1124.9 & 1511.9 & 3.7737 & 2.0447 & 8.9721 & 19.397 & 4.6997 \\
\hline 5 & -0.0020 & 0.0450 & 2.904 & 1083.8 & 1465 & 3.7934 & 1.6842 & 9.3842 & 18.735 & 5.5927 \\
\hline 6 & -0.0016 & 0.0454 & 2.8232 & 1099.8 & 1474.4 & 3.7414 & 1.849 & 8.0205 & 19.899 & 5.2631 \\
\hline 7 & -0.0016 & 0.0463 & 2.7588 & 1092.5 & 1457.1 & 3.7846 & 1.5508 & 8.9071 & 19.252 & 5.9994 \\
\hline 8 & -0.0020 & 0.0451 & 2.7163 & 1100.2 & 1461.9 & 3.7018 & 1.4852 & 8.1622 & 20.417 & 4.457 \\
\hline 9 & -0.0019 & 0.0464 & 2.7459 & 1097.1 & 1459.3 & 3.7545 & 1.6455 & 7.6253 & 22.406 & 4.5061 \\
\hline 10 & -0.0020 & 0.0452 & 2.7131 & 1064.5 & 1412.2 & 3.5901 & 1.2887 & 9.374 & 19.036 & 3.8459 \\
\hline 11 & -0.0017 & 0.0461 & 2.7201 & 1076.2 & 1425.3 & 3.6759 & 1.6932 & 9.0578 & 20.679 & 4.7048 \\
\hline 12 & -0.0015 & 0.0456 & 2.8312 & 1085.7 & 1439.5 & 3.7056 & 1.3783 & 7.0055 & 21.292 & 5.7499 \\
\hline 13 & -0.0024 & 0.0455 & 2.7019 & 1084.2 & 1443.5 & 3.6366 & 1.4298 & 7.8123 & 20.856 & 4.8974 \\
\hline 14 & -0.0010 & 0.0458 & 2.8815 & 1114.1 & 1476 & 3.7454 & 1.8362 & 8.809 & 20.021 & 4.9207 \\
\hline 15 & -0.0023 & 0.0464 & 2.673 & 1086.4 & 1448.9 & 3.7169 & 1.5678 & 7.0345 & 22.417 & 5.7741 \\
\hline 16 & -0.0015 & 0.0456 & 2.8445 & 1093 & 1464.7 & 3.7109 & 1.4436 & 6.2753 & 21.639 & 5.8342 \\
\hline 17 & -0.0020 & 0.0460 & 2.7972 & 1072.2 & 1415.8 & 3.6375 & 1.4832 & 9.8345 & 19.733 & 4.19 \\
\hline 18 & -0.0016 & 0.0459 & 2.7316 & 1065.1 & 1415.7 & 3.6443 & 1.5549 & 9.4308 & 19.652 & 4.6853 \\
\hline 19 & -0.0027 & 0.0467 & 2.6466 & 1074.4 & 1422.4 & 3.6713 & 1.5905 & 9.2169 & 20.213 & 4.994 \\
\hline 20 & -0.0022 & 0.0462 & 2.8096 & 1086.8 & 1440.8 & 3.755 & 1.7385 & 9.0918 & 19.174 & 5.4398 \\
\hline 21 & -0.0014 & 0.0460 & 2.7279 & 1076.6 & 1432 & 3.7479 & 1.4753 & 6.2085 & 22.214 & 5.1469 \\
\hline 22 & -0.0018 & 0.0461 & 2.8124 & 1060.7 & 1421.7 & 3.7194 & 1.523 & 7.1764 & 21.749 & 4.9088 \\
\hline 23 & -0.0018 & 0.04621 & 2.7699 & 1076.6 & 1449.6 & 3.7643 & 1.5759 & 6.3511 & 21.832 & 5.1766 \\
\hline 24 & -0.0022 & 0.0454 & 2.8573 & 1087.6 & 1469.2 & 3.7647 & 1.5709 & 7.9958 & 19.243 & 5.5585 \\
\hline 25 & -0.0019 & 0.0471 & 2.7946 & 1057.5 & 1434.7 & 3.7989 & 1.6254 & 5.8172 & 23.342 & 5.8649 \\
\hline 26 & -0.0021 & 0.0452 & 2.7171 & 1048.3 & 1421.4 & 3.6266 & 1.6351 & 7.578 & 18.711 & 3.7897 \\
\hline 27 & -0.0015 & 0.0455 & 2.6652 & 1068.9 & 1428.3 & 3.6984 & 1.4681 & 7.601 & 21.644 & 4.2268 \\
\hline 28 & -0.0022 & 0.0463 & 2.7377 & 1042.7 & 1392.9 & 3.6335 & 1.3731 & 7.2178 & 20.923 & 5.395 \\
\hline 29 & -0.0023 & 0.0468 & 2.6593 & 1033.1 & 1400.7 & 3.7300 & 1.3639 & 8.9799 & 21.367 & 4.476 \\
\hline 30 & -0.0024 & 0.0458 & 2.7841 & 1063.2 & 1438.5 & 3.7285 & 1.5706 & 6.7465 & 21.867 & 4.3497 \\
\hline$\ldots$ & $\ldots$ & $\ldots$ & $\ldots$ & $\ldots$ & $\ldots$ & $\ldots$ & $\ldots$ & $\ldots$ & $\ldots$ & $\ldots$ \\
\hline 721 & -0.0027 & 0.0530 & 3.1491 & 1656.5 & 2201.3 & 4.9816 & 8.1026 & 14.202 & 22.892 & 8.0045 \\
\hline 722 & -0.0021 & 0.0528 & 3.0908 & 1641 & 2192.9 & 4.9686 & 7.7509 & 11.637 & 24.213 & 8.4416 \\
\hline 723 & -0.0021 & 0.0532 & 3.197 & 1643.1 & 2182.1 & 4.9455 & 7.8388 & 13.55 & 23.99 & 8.3561 \\
\hline 724 & -0.0021 & 0.0544 & 3.1498 & 1593.5 & 2131.6 & 5.0232 & 7.9186 & 12.452 & 26.366 & 6.6946 \\
\hline 725 & -0.0022 & 0.0538 & 3.3512 & 1644.6 & 2172.7 & 4.9371 & 8.0091 & 12.501 & 24.489 & 8.3043 \\
\hline 726 & -0.0026 & 0.0540 & 3.2107 & 1637.6 & 2179.1 & 5.0231 & 7.8501 & 12.894 & 24.837 & 9.0401 \\
\hline 727 & -0.0021 & 0.0534 & 3.1239 & 1656 & 2201.2 & 4.9376 & 8.8428 & 12.423 & 24.195 & 6.4097 \\
\hline 728 & -0.0015 & 0.0540 & 3.1796 & 1650.7 & 2180 & 4.979 & 7.7268 & 13.344 & 22.71 & 9.5417 \\
\hline 729 & -0.0023 & 0.0534 & 3.1097 & 1621.9 & 2164.1 & 4.9523 & 7.7175 & 13.518 & 24.363 & 6.6699 \\
\hline 730 & -0.0019 & 0.0549 & 3.271 & 1595.3 & 2125.1 & 5.032 & 6.8475 & 12.85 & 27.136 & 8.3782 \\
\hline$\ldots$ & $\ldots$ & $\ldots$ & $\ldots$ & $\ldots$ & $\ldots$ & $\ldots$ & $\ldots$ & $\ldots$ & $\ldots$ & $\ldots$ \\
\hline 965 & -0.0009 & 0.0991 & 3.0364 & 1487.9 & 2097.5 & 9.54 & 21.225 & 32.315 & 82.163 & 37.627 \\
\hline 966 & -0.0006 & 0.1268 & 2.8109 & 1515.1 & 1937.2 & 10.976 & 27.715 & 92.465 & 140.51 & 40.1 \\
\hline 967 & -0.0019 & 0.1162 & 2.8857 & 1421.4 & 1958.3 & 10.576 & 27.599 & 28.219 & 161.65 & 27.7 \\
\hline 968 & -0.0008 & 0.1139 & 2.5798 & 1555.5 & 2121.7 & 10.546 & 34.988 & 40.41 & 134.55 & 36.982 \\
\hline 969 & -0.0019 & 0.1175 & 2.4147 & 1624.7 & 2152.2 & 10.742 & 33.982 & 84.171 & 82.756 & 49.921 \\
\hline 970 & -0.0006 & 0.1346 & 3.3694 & 1529.6 & 2086.0 & 13.284 & 39.086 & 113.03 & 126.62 & 56.488 \\
\hline
\end{tabular}


TABLE 2: Continued.

\begin{tabular}{lcccccccccc}
\hline Num & $\bar{Y}$ & $Y_{\mathrm{RMS}}$ & $\mathrm{K}_{V}$ & $F_{C}$ & $\mathrm{RMSF}$ & $\mathrm{RVF}$ & $E\left[\mathrm{IMF}_{1}\right]$ & $E\left[\mathrm{IMF}_{2}\right]$ & $E\left[\mathrm{IMF}_{3}\right]$ & $E\left[\mathrm{IMF}_{4}\right]$ \\
\hline 971 & -0.0030 & 0.1604 & 3.2912 & 1541.5 & 2029.4 & 15.441 & 48.641 & 148.64 & 170.37 & 84.156 \\
972 & -0.0021 & 0.1741 & 5.6245 & 1529.0 & 2118.3 & 17.824 & 63.52 & 77.414 & 191.18 & 126.03 \\
973 & -0.0022 & 0.1537 & 3.9313 & 1676.3 & 2168.1 & 14.944 & 57.251 & 190.61 & 137.94 & 62.819 \\
974 & -0.0023 & 0.1478 & 9.198 & 1712.6 & 2278 & 16.219 & 48.644 & 99.237 & 93.873 & 86.746 \\
975 & -0.0024 & 0.1931 & 3.8436 & 1721.9 & 2111.3 & 17.819 & 72.926 & 400.09 & 178.84 & 64.739 \\
976 & 0.0033 & 0.3194 & 13.925 & 1586.2 & 2060.7 & 31.139 & 175.56 & 276.13 & 498.11 & 459.64 \\
977 & -0.0052 & 0.2257 & 12.091 & 1587.2 & 2047.1 & 22.023 & 88.389 & 245.69 & 171.25 & 209.03 \\
978 & -0.0032 & 0.1931 & 6.1532 & 1688.3 & 2100.0 & 17.47 & 64.441 & 349.32 & 160.57 & 114.69 \\
979 & -0.0003 & 0.1467 & 4.3359 & 1568.5 & 2057.8 & 14.038 & 43.377 & 90.334 & 178.96 & 84.912 \\
980 & -0.0027 & 0.3737 & 13.955 & 1515.8 & 1912.7 & 32.214 & 177.87 & 832.19 & 504.77 & 552.48 \\
981 & -0.0044 & 0.2026 & 8.5665 & 1535 & 2008 & 19.342 & 64.232 & 200.84 & 197.54 & 175.44 \\
982 & -0.0016 & 0.2355 & 10.819 & 1473.8 & 1907.3 & 20.928 & 97.985 & 323.58 & 299.29 & 201.22 \\
983 & 0.0018 & 0.0019 & 1.4515 & 67.2 & 464.93 & 0.1035 & 0.0003 & 0.0008 & 0.0010 & 0.0009 \\
984 & -0.0011 & 0.0014 & 1.7458 & 104.61 & 492.94 & 0.07541 & 0.0002 & 0.0004 & 0.0006 & 0.0010 \\
\hline
\end{tabular}

\section{Verification Experiment}

In order to verify the validity of the principal component extraction method for vibration signal feature parameters of $\mathrm{R} 2 \mathrm{R}$ processing roll for flexible material, an experimental platform for flexible material roll-to-roll processing was built as shown in Figure 2. The experimental platform was mainly composed of mechanical structure and driving control unit. The mechanical structure used standard reinforced aluminum alloy profiles as the support frame, including unwinding module, driving module, and rewinding module. The unwinding, rewinding, and driving module all adopt $120 \mathrm{~W}$ speed regulating motor; the driving module adopts counterroll mode, and the roll rotates under the motor driving. The upper roll was controlled by a hand-operated lifting handle. The upper rubber-covered roll should be lifted to make the material go around the upper rubber-covered roll and then flat the material. After the correct alignment, the upper rubber-covered roll should be put down, and the upper and lower roll rotates simultaneously to realize the material transmission. The adaptive maximum width of experimental platform is $450 \mathrm{~mm}$, which can be used to transport material thickness of 0.1-5 mm. Blue PET polyester film with a width of $50 \mathrm{~mm}$, a thickness of $0.05 \mathrm{~mm}$, a density of $1450 \mathrm{~kg} / \mathrm{m}^{3}$, an elastic modulus of $3495 \mathrm{MPa}$, and a Poisson's ratio of 0.3 was used in the experiment. The magnet adsorption triaxial accelerometer was selected to realize data acquisition. The sensitivity is $20 \mathrm{mv} / \mathrm{g}$, the frequency response is $5-5000 \mathrm{~Hz}$, and the size is $24 * 24 * 15 \mathrm{~mm}$. The adsorption installation method will not destroy the measured object, so it was conve- nient to move. In addition to the hardware mentioned above, the experimental platform also includes a professional version of the Lenovo desktop computer in Windows 7 and software data processing software MATLAB R2014a.

Considering that the driving module is the core of the whole experimental platform as well as the roll and the bearing are closely connected, the vibration of the roll will be fully reflected in the bearing, so the three-axis accelerometer was adsorbed on the bearing seat on one side of the driving roll to realize data acquisition of vibration signal. The sampling frequency is $10 \mathrm{kHz}$, data acquisition was conducted every 60 minutes, each time for $1 \mathrm{~s}$, and a total of 984 groups of samples were collected. With the gradual progress of the experiment, performance of driving rolls declines in varying degrees.

(1) Sample data denoising processing. According to equation (1), sliding average filtering method $y_{L}=\left(x_{L}+x_{\mathrm{L}-1}+x_{\mathrm{L}-2}+\cdots\right.$ $\left.+x_{\mathrm{L}-\mathrm{M}-1}\right) / M$, the sliding window width $M$ was 5 , input 984 sets of sample data for calculation. By using the sliding smoothing algorithm, the original sample data was further smoothed and denoised which means the systematic errors and random errors of the sample data were finally eliminated and reduced. Figure 3 is a comparison of the effects before and after noise reduction of partial data of the first group of samples.

(2) Constructed the original feature parameter matrix $Z_{\text {org }}$ . Substitute the noise-reduced sample data into the original feature parameter matrix $Z_{\text {org }}$ of flexible material $\mathrm{R} 2 \mathrm{R}$ processing roller vibration signal of Section 1 (see equation (3)), shown in the equation below

$$
Z_{\text {org }}=\left(\begin{array}{cccccccccc}
\bar{Y}_{1} & Y_{\mathrm{RMS} 1} & \mathrm{~K}_{V_{1}} & F_{C_{1}} & \mathrm{RMSF}_{1} & \mathrm{RVF}_{1} & E\left[\mathrm{IMF}_{1}\right]_{1} & E\left[\mathrm{IMF}_{2}\right]_{1} & E\left[\mathrm{IMF}_{3}\right]_{1} & E\left[\mathrm{IMF}_{4}\right]_{1} \\
\bar{Y}_{2} & Y_{\mathrm{RMS} 2} & \mathrm{~K}_{V_{2}} & F_{C_{2}} & \mathrm{RMSF}_{2} & \mathrm{RVF}_{2} & E\left[\mathrm{IMF}_{1}\right]_{2} & E\left[\mathrm{IMF}_{2}\right]_{2} & E\left[\mathrm{IMF}_{3}\right]_{2} & E\left[\mathrm{IMF}_{4}\right]_{2} \\
\vdots & \vdots & \vdots & \vdots & \vdots & \vdots & \vdots & \vdots & \vdots & \vdots \\
\bar{Y}_{n} & Y_{\mathrm{RMS} n} & \mathrm{~K}_{V_{n}} & F_{C_{n}} & \mathrm{RMSF}_{n} & \mathrm{RVF}_{n} & E\left[\mathrm{IMF}_{1}\right]_{n} & E\left[\mathrm{IMF}_{2}\right]_{n} & E\left[\mathrm{IMF}_{3}\right]_{n} & E\left[\mathrm{IMF}_{4}\right]_{n}
\end{array}\right)
$$


TABLE 3: Normalized feature parameter matrix of roller vibration signal of flexible material R2R processing.

\begin{tabular}{|c|c|c|c|c|c|c|c|c|c|c|}
\hline Num & $\bar{Y}$ & $Y_{\mathrm{RMS}}$ & $\mathrm{K}_{V}$ & $F_{C}$ & RMSF & RVF & $E\left[\mathrm{IMF}_{1}\right]$ & $E\left[\mathrm{IMF}_{2}\right]$ & $E\left[\mathrm{IMF}_{3}\right]$ & $E\left[\mathrm{IMF}_{4}\right]$ \\
\hline 1 & 5.24780 & 0.85109 & 0.95367 & 0.87938 & 0.91096 & 0.83445 & 0.35141 & 0.50069 & 0.65705 & 0.48691 \\
\hline 2 & 1.33210 & 0.83235 & 1.00790 & 0.91329 & 0.94204 & 0.84923 & 0.33286 & 0.41796 & 0.65118 & 0.57744 \\
\hline 3 & 1.27590 & 0.85543 & 0.92999 & 0.9096 & 0.92616 & 0.85343 & 0.36774 & 0.42606 & 0.70683 & 0.54777 \\
\hline 4 & 1.17040 & 0.86733 & 0.96239 & 0.94184 & 0.94926 & 0.85925 & 0.46489 & 0.58723 & 0.69018 & 0.5304 \\
\hline 5 & 1.23780 & 0.86618 & 0.99268 & 0.90743 & 0.91982 & 0.86374 & 0.38293 & 0.6142 & 0.66662 & 0.63119 \\
\hline 6 & 0.83498 & 0.86318 & 0.96506 & 0.92082 & 0.92572 & 0.85189 & 0.4204 & 0.52494 & 0.70804 & 0.59399 \\
\hline 7 & 0.82787 & 0.87967 & 0.94304 & 0.91471 & 0.91486 & 0.86173 & 0.3526 & 0.58297 & 0.68502 & 0.67709 \\
\hline 8 & 1.04540 & 0.85718 & 0.92852 & 0.92116 & 0.91787 & 0.84288 & 0.33768 & 0.53422 & 0.72647 & 0.50301 \\
\hline 9 & 0.98681 & 0.88172 & 0.93863 & 0.91856 & 0.91624 & 0.85487 & 0.37413 & 0.49908 & 0.79724 & 0.50855 \\
\hline 10 & 1.07670 & 0.85809 & 0.92742 & 0.89127 & 0.88667 & 0.81744 & 0.293 & 0.61353 & 0.67733 & 0.43404 \\
\hline 11 & 0.92484 & 0.87578 & 0.92981 & 0.90106 & 0.89489 & 0.83697 & 0.38497 & 0.59284 & 0.73579 & 0.53098 \\
\hline 12 & 0.80199 & 0.86565 & 0.96779 & 0.90902 & 0.90381 & 0.84375 & 0.31338 & 0.45851 & 0.7576 & 0.64893 \\
\hline 13 & 1.26200 & 0.86441 & 0.92359 & 0.90776 & 0.90632 & 0.82802 & 0.32509 & 0.51132 & 0.74209 & 0.55272 \\
\hline 14 & 0.53033 & 0.8703 & 0.98499 & 0.9328 & 0.92672 & 0.85279 & 0.41749 & 0.57655 & 0.71238 & 0.55535 \\
\hline 15 & 1.21920 & 0.88188 & 0.91371 & 0.9096 & 0.90971 & 0.84631 & 0.35646 & 0.46041 & 0.79763 & 0.65166 \\
\hline 16 & 0.78526 & 0.8673 & 0.97234 & 0.91513 & 0.91963 & 0.84494 & 0.32822 & 0.41072 & 0.76995 & 0.65844 \\
\hline 17 & 1.03830 & 0.87362 & 0.95617 & 0.89772 & 0.88893 & 0.82824 & 0.33723 & 0.64367 & 0.70213 & 0.47288 \\
\hline 18 & 0.85757 & 0.87202 & 0.93375 & 0.89177 & 0.88886 & 0.82978 & 0.35353 & 0.61725 & 0.69925 & 0.52878 \\
\hline 19 & 1.41970 & 0.88696 & 0.90469 & 0.89956 & 0.89307 & 0.83592 & 0.36162 & 0.60325 & 0.71921 & 0.56362 \\
\hline 20 & 1.16230 & 0.87736 & 0.96041 & 0.90994 & 0.90462 & 0.85499 & 0.39527 & 0.59506 & 0.68224 & 0.61393 \\
\hline 21 & 0.74434 & 0.87358 & 0.93248 & 0.9014 & 0.8991 & 0.85338 & 0.33543 & 0.40635 & 0.79041 & 0.58087 \\
\hline 22 & 0.93086 & 0.87694 & 0.96137 & 0.88809 & 0.89263 & 0.84688 & 0.34628 & 0.4697 & 0.77386 & 0.5540 \\
\hline 23 & 0.93045 & 0.87721 & 0.94684 & 0.9014 & 0.91015 & 0.8571 & 0.3583 & 0.41568 & 0.77682 & 0.58423 \\
\hline 24 & 1.15700 & 0.86185 & 0.97671 & 0.91061 & 0.92245 & 0.85719 & 0.35717 & 0.52333 & 0.6847 & 0.62733 \\
\hline 25 & 1.01660 & 0.89488 & 0.95528 & 0.88541 & 0.90079 & 0.86499 & 0.36956 & 0.38074 & 0.83055 & 0.66191 \\
\hline 26 & 1.11800 & 0.85819 & 0.92879 & 0.8777 & 0.89244 & 0.82575 & 0.37176 & 0.49598 & 0.66577 & 0.4277 \\
\hline 27 & 0.78011 & 0.86437 & 0.91105 & 0.89495 & 0.89677 & 0.84211 & 0.33379 & 0.49749 & 0.77013 & 0.47703 \\
\hline 28 & 1.15440 & 0.87994 & 0.93583 & 0.87302 & 0.87455 & 0.82733 & 0.31219 & 0.47241 & 0.74447 & 0.60887 \\
\hline 29 & 1.21180 & 0.88856 & 0.90903 & 0.86498 & 0.87945 & 0.84929 & 0.3101 & 0.58774 & 0.76027 & 0.50516 \\
\hline 30 & 1.25660 & 0.86975 & 0.95169 & 0.89018 & 0.90318 & 0.84895 & 0.3571 & 0.44156 & 0.77806 & 0.4909 \\
\hline$\ldots$ & $\ldots$ & $\ldots$ & ... & $\ldots$ & $\cdots$ & $\cdots$ & $\ldots$ & $\ldots$ & $\cdots$ & $\cdots$ \\
\hline 721 & 1.42710 & 1.00740 & 1.07650 & 1.38690 & 1.38210 & 1.13430 & 1.84220 & 0.92952 & 0.81453 & 0.90338 \\
\hline 722 & 1.11540 & 1.00290 & 1.05650 & 1.37400 & 1.37680 & 1.13130 & 1.76230 & 0.76164 & 0.86154 & 0.95271 \\
\hline 723 & 1.09110 & 1.01050 & 1.09280 & 1.37570 & 1.37010 & 1.12610 & 1.78230 & 0.88685 & 0.8536 & 0.94306 \\
\hline 724 & 1.09370 & 1.03290 & 1.07670 & 1.33420 & 1.33830 & 1.14380 & 1.80040 & 0.81499 & 0.93815 & 0.75555 \\
\hline 725 & 1.16310 & 1.02140 & 1.14550 & 1.37700 & 1.36420 & 1.12420 & 1.82100 & 0.81819 & 0.87136 & 0.93722 \\
\hline 726 & 1.33680 & 1.02560 & 1.09750 & 1.37110 & 1.36820 & 1.14370 & 1.78480 & 0.84392 & 0.88374 & 1.0203 \\
\hline 727 & 1.10150 & 1.01420 & 1.06780 & 1.38650 & 1.38200 & 1.12430 & 2.01050 & 0.81309 & 0.8609 & 0.72339 \\
\hline 728 & 0.80770 & 1.02520 & 1.08690 & 1.38210 & 1.36870 & 1.13370 & 1.75680 & 0.87337 & 0.80806 & 1.0769 \\
\hline 729 & 1.18380 & 1.01510 & 1.06300 & 1.35800 & 1.35880 & 1.12760 & 1.75470 & 0.88476 & 0.86688 & 0.75276 \\
\hline 730 & 1.0100 & 1.02530 & 1.08540 & 1.36890 & 1.36230 & 1.13010 & 1.81890 & 0.8505 & 0.8628 & 0.86240 \\
\hline$\ldots$ & $\ldots$ & $\ldots$ & $\ldots$ & $\ldots$ & $\ldots$ & $\ldots$ & $\ldots$ & $\ldots$ & $\ldots$ & $\ldots$ \\
\hline 965 & 0.47103 & 1.88220 & 1.03790 & 1.24580 & 1.31690 & 2.17220 & 4.82580 & 2.11500 & 2.92350 & 4.246500 \\
\hline 966 & 0.31825 & 2.40740 & 0.96085 & 1.26850 & 1.21630 & 2.49920 & 6.30140 & 6.05190 & 4.99960 & 4.52560 \\
\hline 967 & 1.01440 & 2.20690 & 0.98642 & 1.19010 & 1.22950 & 2.40810 & 6.27500 & 1.84690 & 5.75180 & 3.12620 \\
\hline 968 & 0.42577 & 2.16310 & 0.88186 & 1.30240 & 1.33210 & 2.40120 & 7.95500 & 2.64480 & 4.78750 & 4.17380 \\
\hline 969 & 0.99849 & 2.23220 & 0.82542 & 1.36030 & 1.35130 & 2.44600 & 7.72630 & 5.50900 & 2.94460 & 5.63400 \\
\hline 970 & 0.32912 & 2.55640 & 1.15180 & 1.28070 & 1.30970 & 3.02470 & 8.88680 & 7.39780 & 4.50530 & 6.37520 \\
\hline
\end{tabular}


TABLe 3: Continued.

\begin{tabular}{lcccccccccc}
\hline Num & $\bar{Y}$ & $Y_{\mathrm{RMS}}$ & $\mathrm{K}_{V}$ & $F_{C}$ & $\mathrm{RMSF}$ & $\mathrm{RVF}$ & $E\left[\mathrm{IMF}_{1}\right]$ & $E\left[\mathrm{IMF}_{2}\right]$ & $E\left[\mathrm{IMF}_{3}\right]$ & $E\left[\mathrm{IMF}_{4}\right]$ \\
\hline 971 & 1.56240 & 3.04540 & 1.12500 & 1.29060 & 1.27420 & 3.51590 & 11.0590 & 9.72850 & 6.0620 & 9.49780 \\
972 & 1.09310 & 3.3060 & 1.92260 & 1.28020 & 1.33000 & 4.05830 & 14.4420 & 5.06680 & 6.80250 & 14.2240 \\
973 & 1.17300 & 2.91760 & 1.34380 & 1.40350 & 1.36130 & 3.40270 & 13.0170 & 12.4750 & 4.90810 & 7.08970 \\
974 & 1.18180 & 2.80640 & 3.14420 & 1.43390 & 1.43030 & 3.69300 & 11.060 & 6.49510 & 3.34020 & 9.79010 \\
975 & 1.25600 & 3.66710 & 1.31390 & 1.44170 & 1.32560 & 4.05720 & 16.5810 & 26.1860 & 6.36340 & 7.30640 \\
976 & -1.74260 & 6.06420 & 4.76000 & 1.32810 & 1.29380 & 7.09030 & 39.9160 & 18.0730 & 17.7240 & 51.8750 \\
977 & 2.70040 & 4.28550 & 4.13310 & 1.32890 & 1.28530 & 5.01450 & 20.0960 & 16.0800 & 6.09340 & 23.5910 \\
978 & 1.64570 & 3.66710 & 2.10340 & 1.41360 & 1.31850 & 3.97790 & 14.6520 & 22.8630 & 5.71330 & 12.9440 \\
979 & 0.18971 & 2.78550 & 1.48210 & 1.31320 & 1.29200 & 3.19650 & 9.86240 & 5.91240 & 6.36077 & 9.58310 \\
980 & 1.42060 & 7.09550 & 4.77030 & 1.26910 & 1.20090 & 7.33490 & 40.4410 & 54.4670 & 17.9610 & 62.3520 \\
981 & 2.31030 & 3.84700 & 2.92830 & 1.28520 & 1.26070 & 4.40410 & 14.6040 & 13.1450 & 7.02880 & 19.8000 \\
982 & 0.86468 & 4.47100 & 3.69830 & 1.23400 & 1.19750 & 4.76520 & 22.2780 & 21.1780 & 10.6490 & 22.7090 \\
983 & -0.95567 & 0.03758 & 0.49617 & 0.05626 & 0.29191 & 0.02356 & 0.00008 & 0.00005 & 0.00003 & 0.00010 \\
984 & 0.59807 & 0.02724 & 0.59677 & 0.08758 & 0.30950 & 0.01717 & 0.00005 & 0.00003 & 0.00002 & 0.00010 \\
\hline
\end{tabular}

The average value, root mean square, kurtosis index, center of gravity frequency, root mean square of the frequency, standard deviation of frequency, and the energy of intrinsic mode function components of sample data were calculated. The calculation results of the above indexes are given in Figures 4-6, respectively, and some calculation results of each feature index are given in Table 2.

The root mean square and kurtosis indicators in Figure 4 have a slight upward trend in the early stage of the fault, and the mutation phenomenon appeared until the 970th sample point. The sensitivity of immediate domain feature to the early fault and medium fault was general, and the fault characterization of late failure phase was relatively strong. In Figure 5, the frequency-domain feature curves of the center of gravity frequency were slightly increased at about 500th sample point and rose sharply at 700 points. Both were in the upper and lower oscillating unstable state in subsequent time period and were significantly mutated at the 970th sample point. The trend of the frequency standard deviation feature curve was like the trend of the time domain feature change. In Figure 6, the energy of different eigenmode functions has a great difference in energy variation when the fault occurs and can characterize the energy distribution of the roller vibration signal in the frequency domain.
TABle 4: Cumulative variance contribution rate table of roll vibration signals for flexible material R2R system.

\begin{tabular}{lccc}
\hline $\begin{array}{l}\text { Eigenvalue } \\
\text { number }\end{array}$ & Eigenvalue & $\begin{array}{c}\text { Variance } \\
\text { ratio }\end{array}$ & $\begin{array}{c}\text { Accumulated } \\
\text { contribution rate }\end{array}$ \\
\hline$\lambda_{1}$ & 21.213 & 0.930 & 0.930 \\
$\lambda_{2}$ & 0.924 & 0.041 & 0.971 \\
$\lambda_{3}$ & 0.427 & 0.019 & 0.989 \\
$\lambda_{4}$ & 0.145 & 0.006 & 0.995 \\
$\lambda_{5}$ & 0.063 & 0.003 & 0.998 \\
$\lambda_{6}$ & 0.027 & 0.001 & 0.999 \\
$\lambda_{7}$ & 0.009 & 0.000 & 1.000 \\
$\lambda_{8}$ & 0.005 & 0.000 & 1.000 \\
$\lambda_{9}$ & 0.001 & 0.000 & 1.000 \\
$\lambda_{10}$ & 0.000 & 0.000 & 1.000 \\
\hline
\end{tabular}

(3) Normalization of the original feature parameter matrix. Substituting the original feature data of Table 2 into the equation (6) of Section 2, as shown below

$$
Z_{\text {org }}{ }^{*}=\left(\begin{array}{cccccccccc}
\bar{Y}_{1}{ }^{*} & Y_{\mathrm{RMS}_{1}}{ }^{*} & \mathrm{~K}_{V 1}{ }^{*} & F_{C_{1}}{ }^{*} & \mathrm{RMSF}_{1}{ }^{*} & \mathrm{RVF}_{1}{ }^{*} & E\left[\mathrm{IMF}_{1}\right]_{1}{ }^{*} & E\left[\mathrm{IMF}_{2}\right]_{1}{ }^{*} & E\left[\mathrm{IMF}_{3}\right]_{1}{ }^{*} & E\left[\mathrm{IMF}_{4}\right]_{1}{ }^{*} \\
\bar{Y}^{*}{ }_{2} & Y_{\mathrm{RMS}_{2}}{ }^{*} & \mathrm{~K}_{V_{2}}{ }^{*} & F_{C_{2}}{ }^{*} & \mathrm{RMSF}_{2}{ }^{*} & \mathrm{RVF}_{2}{ }^{*} & E\left[\mathrm{IMF}_{1}\right]_{2}{ }^{*} & E\left[\mathrm{IMF}_{2}\right]_{2}{ }^{*} & E\left[\mathrm{IMF}_{3}\right]_{2}{ }^{*} & E\left[\mathrm{IMF}_{4}\right]_{2}{ }^{*} \\
\vdots & \vdots & \vdots & \vdots & \vdots & \vdots & \vdots & \vdots & \vdots & \vdots \\
\bar{Y}_{n}{ }^{*} & Y_{\mathrm{RMS}_{n}}{ }^{*} & \mathrm{~K}_{V n}{ }^{*} & F_{C_{n}}{ }^{*} & \mathrm{RMSF}_{n}{ }^{*} & \mathrm{RVF}_{n}{ }^{*} & E\left[\mathrm{IMF}_{1}\right]_{n}{ }^{*} & E\left[\mathrm{IMF}_{2}\right]_{n}{ }^{*} & E\left[\mathrm{IMF}_{3}\right]_{n}{ }^{*} & E\left[\mathrm{IMF}_{4}\right]_{n}{ }^{*}
\end{array}\right) .
$$


The original feature matrix was normalized and calculated, and the normalized average value, root mean square, kurtosis index, center of gravity frequency, frequency root mean square, frequency standard deviation, and energy of intrinsic mode functions component were obtained, as shown in Table 3.

(4) Constructing the covariance matrix of normalized feature parameters. On the basis of normalized processing of $Z_{\text {org }}$ feature parameter matrix, the normalized feature parameter matrix of roller vibration signals processed by R2R flexible material in Table 3 is divided into columns and substituted into Section 3.3 equation (7) as follows

$$
C_{Z}=\left[\begin{array}{ccc}
\operatorname{cov}\left(\bar{Y}^{*}, \bar{Y}^{*}\right) & \cdots & \operatorname{cov}\left(\bar{Y}^{*}, E\left[\mathrm{IMF}_{4}\right]^{*}\right) \\
\vdots & \ddots & \vdots \\
\operatorname{cov}\left(E\left[\mathrm{IMF}_{4}\right]^{*}, \bar{Y}^{*}\right) & \cdots & \operatorname{cov}\left(E\left[\mathrm{IMF}_{4}\right]^{*}, E\left[\mathrm{IMF}_{4}\right]^{*}\right)
\end{array}\right] .
$$

The covariance matrix of the feature parameters was calculated.

$$
C_{Z}^{\prime}=\left[\begin{array}{llllllllll}
0.0664 & -0.0022 & 0.0003 & 0.0009 & 0.0006 & -0.0031 & -0.0445 & 0.0278 & -0.0283 & -0.0557 \\
-0.0022 & 0.1951 & 0.0879 & 0.0315 & 0.0249 & 0.2150 & 1.0156 & 0.9626 & 0.4895 & 1.1391 \\
0.0003 & 0.0879 & 0.0610 & 0.0135 & 0.0119 & 0.1021 & 0.5413 & 0.4774 & 0.2188 & 0.6696 \\
0.0009 & 0.0315 & 0.0135 & 0.0210 & 0.0188 & 0.0384 & 0.1515 & 0.1209 & 0.0610 & 0.1094 \\
0.0006 & 0.0249 & 0.0119 & 0.0188 & 0.0176 & 0.0323 & 0.1298 & 0.0938 & 0.0466 & 0.0913 \\
-0.0031 & 0.2150 & 0.1021 & 0.0384 & 0.0323 & 0.2432 & 1.1529 & 1.0534 & 0.5343 & 1.2796 \\
-0.0445 & 1.0156 & 0.5413 & 0.1515 & 0.1298 & 1.1529 & 5.9754 & 5.4208 & 2.6112 & 6.9105 \\
0.0278 & 0.9626 & 0.4774 & 0.1209 & 0.0938 & 1.0534 & 5.4208 & 6.0386 & 2.4058 & 6.4036 \\
-0.0283 & 0.4895 & 0.2188 & 0.0610 & 0.0466 & 0.5343 & 2.6112 & 2.4058 & 1.3060 & 3.0370 \\
-0.0557 & 1.1391 & 0.6696 & 0.1094 & 0.0913 & 1.2796 & 6.9105 & 6.4036 & 3.0370 & 8.8900
\end{array} .\right.
$$

(5) Solving the eigenvalues and eigenvectors of the covariance matrix. According to $\left|c^{(k-1)}{ }_{p q}\right|=\max \left|c_{i \neq j}^{(k-1)}{ }_{i j}\right|$ in Section 3.3, $p$

In the experiment, the number of orthogonal transforma-
, $q$, and $R_{p q}(\theta)$ were determined, and the covariance matrix $C_{Z}^{\prime}$ was transformed orthogonally according to the equation

$$
C_{Z}{ }^{(k)}=R_{p q}^{(k)_{T}}(\theta) C_{Z}^{(k-1)} R_{p q}^{(k)}(\theta)=R_{p q}^{(k)_{T}} R_{p q}^{(k-1)_{T}} \cdots R_{p q}^{(1)_{T}} C_{Z}{ }^{(0)} R_{p q}^{(1)}(\theta) \cdots R_{p q}^{(k-1)}(\theta) R_{p q}^{(k)}(\theta)
$$

tions is $k=18$. The eigenvalue of the covariance matrix was calculated as follows:

$$
C_{Z}^{(18)}=\left[\begin{array}{cccccccccc}
21.213 & 0 & 0 & 0 & 0 & 0 & 0 & 0 & 0 & 0 \\
0 & 0.924 & 0 & 0 & 0 & 0 & 0 & 0 & 0 & 0 \\
0 & 0 & 0.472 & 0 & 0 & 0 & 0 & 0 & 0 & 0 \\
0 & 0 & 0 & 0.145 & 0 & 0 & 0 & 0 & 0 & 0 \\
0 & 0 & 0 & 0 & 0.063 & 0 & 0 & 0 & 0 & 0 \\
0 & 0 & 0 & 0 & 0 & 0.027 & 0 & 0 & 0 & 0 \\
0 & 0 & 0 & 0 & 0 & 0 & 0.009 & 0 & 0 & 0 \\
0 & 0 & 0 & 0 & 0 & 0 & 0 & 0.005 & 0 & 0 \\
0 & 0 & 0 & 0 & 0 & 0 & 0 & 0 & 0.001 & 0 \\
0 & 0 & 0 & 0 & 0 & 0 & 0 & 0 & 0 & 0.0000936
\end{array}\right]
$$


Furthermore, the eigenvectors corresponding to the 10 eigenvalues on the diagonal line of the above eigenvalue matrix $C_{Z}{ }^{(18)}$ were calculated, respectively. Finally, the eigen- vectors corresponding to all eigenvalues were obtained as shown in matrix $U^{\prime}$.

$$
U^{\prime}=\left[\begin{array}{cccccccccc}
-0.002 & -0.069 & 0.053 & 0.071 & 0.965 & -0.207 & 0.116 & 0.001 & -0.002 & -0.005 \\
0.089 & -0.040 & -0.188 & -0.177 & 0.129 & 0.277 & -0.378 & 0.203 & 0.743 & 0.307 \\
0.048 & 0.048 & 0.009 & 0.137 & 0.096 & 0.191 & -0.509 & -0.813 & -0.099 & 0.017 \\
0.011 & -0.024 & -0.115 & 0.102 & 0.070 & 0.575 & 0.400 & -0.137 & 0.265 & -0.625 \\
0.009 & -0.013 & -0.101 & 0.126 & 0.060 & 0.516 & 0.406 & -0.060 & -0.259 & 0.684 \\
0.100 & -0.019 & -0.231 & -0.054 & 0.153 & 0.389 & -0.470 & 0.457 & -0.531 & -0.215 \\
0.521 & 0.109 & -0.612 & 0.510 & -0.059 & -0.269 & 0.057 & 0.005 & 0.047 & 0.003 \\
0.504 & -0.817 & 0.267 & -0.016 & -0.068 & 0.018 & 0.008 & -0.024 & -0.024 & -0.003 \\
0.231 & 0.028 & -0.421 & -0.804 & 0.041 & -0.103 & 0.184 & -0.246 & -0.123 & -0.021 \\
0.633 & 0.556 & 0.511 & -0.094 & 0.040 & 0.112 & 0.033 & 0.069 & 0.011 & -0.002
\end{array}\right] .
$$

Then, the 10 eigenvalues on the diagonal line of the above eigenvalue matrix $C_{Z}{ }^{(18)}$ were substituted into $\lambda_{i}$ / $\sum_{i=1}^{m} \lambda_{i}, m=10$ to calculate the variance ratio (principal component value of a single eigenvalue). The cumulative contribution rate of the eigenvalues was calculated by substituting 10 eigenvalues on the diagonal line of the above eigenvalue matrix $C_{Z}{ }^{(18)}$ into $\eta=\left(\sum_{i=1}^{l} \lambda_{i}\right) /\left(\sum_{i=1}^{m} \lambda_{i}\right)$, $l=[1,2, \cdots, 10], m=10$. The calculation results of the variance ratio and the cumulative contribution ratio are shown in Table 4.

From Table 4, it can be seen that the variance ratios of eigenvalues $\lambda_{1}, \lambda_{2}, \lambda_{3}, \lambda_{4}, \lambda_{5}$, and $\lambda_{6}$ are $0.930,0.041,0.019$, $0.006,0.003$, and 0.001 , respectively, while the variance ratios of eigenvalues $\lambda_{7}, \lambda_{8}, \lambda_{9}$, and $\lambda_{10}$ are close to 0.000 , which indicates that $\lambda_{7}, \lambda_{8}, \lambda_{9}$, and $\lambda_{10}$ contain almost no feature information of vibration signals and can be regarded as secondary variables. Similarly, the variance ratio of the eigenvalues $\lambda_{5}$ and $\lambda_{6}$ is less than 0.005 . Compared with the eigenvalues $\lambda_{1}, \lambda_{2}, \lambda_{3}$, and $\lambda_{4}$, it can also be considered as a secondary variable. So $\lambda_{1}, \lambda_{2}, \lambda_{3}$, and $\lambda_{4}$ were selected to calculate the principal component.

(6) Constructing PCA principal component performance degradation index and draw three-dimensional contour map. The normalized feature parameter matrix (see Table 3) and the abovementioned eigenvector matrix $U^{\prime}$ of vibration signal of the $\mathrm{R} 2 \mathrm{R}$ processing roll for flexible material were used to calculate the principal component of vibration signal feature parameters by equation (19), and the results are shown in Table 5 (part)

$$
\left\{\begin{array}{l}
F_{1}=u_{11} \bar{Y}^{*}{ }_{1}+u_{21} Y_{\mathrm{RMS}_{1}}{ }^{*}+\cdots+u_{r 1} E\left[\mathrm{IMF}_{4}\right]_{1}{ }^{*}, \\
F_{2}=u_{12} \bar{Y}^{*}{ }_{2}+u_{22} Y_{\mathrm{RMS}_{2}}{ }^{*}+\cdots+u_{r 2} E\left[\mathrm{IMF}_{4}\right]_{2}{ }^{*}, \\
\vdots \\
F_{l}=u_{1 l} \bar{Y}^{*}{ }_{n}+u_{2 l} Y_{\mathrm{RMS}}{ }_{n}+\cdots+u_{r l} E\left[\mathrm{IMF}_{4}\right]_{n}{ }^{*} .
\end{array}\right.
$$

For the accuracy of performance degradation assessment, the selection of principal component requires that the dimension of principal component is minimum to reduce the computational complexity of the principal component analysis process while the original signal feature information is included. According to Table 4, the variance ratio of $\lambda_{1}$ is 0.930. In principle, only the principal component $F_{1}$ is selected to characterize the decline of the performance of the roll shafts for flexible material R2R processing. The performance declining curve of principal component $F_{1}$ which plotted according to the calculation results in Table 5 is shown in Figure 7. The performance declining curve of principal component $F_{1}$ is plotted as shown in Figure 7.

As can be seen from Figure 7, the changing trend of principal component $F_{1}$ tends to be stable in the early stage (near 500 sample points) and the middle stage (near 700 sample points) of the performance decline, but it does not show a significant upward trend until the late stage (near 900 sample points) of the performance decline, which cannot clearly distinguish the early performance decline from the midperformance decline of the roll shaft. To this end, the performance degradation curve of the principal components $F_{1}, F_{2}, F_{3}$, and , $F_{4}$ corresponding to eigenvalues $\lambda_{1}, \lambda_{2}, \lambda_{3}$, and $\lambda_{4}$ was drawn, as shown in Figure 8. 
TABle 5: Principal component calculation of feature parameters of vibration signals (part).

\begin{tabular}{|c|c|c|c|c|c|c|c|c|c|}
\hline Num & $F_{1}$ & $F_{2}$ & $F_{3}$ & $F_{4}$ & Num & $F_{1}$ & $F_{2}$ & $F_{3}$ & $F_{4}$ \\
\hline 1 & -1.03820 & 0.24010 & -0.45960 & -0.30863 & 701 & -0.66965 & -0.066206 & 0.059745 & -0.17043 \\
\hline 2 & -1.02110 & -0.14789 & -0.28357 & -0.0355 & 702 & -0.58086 & -0.094848 & 0.083001 & -0.24488 \\
\hline 3 & -1.0060 & -0.12953 & -0.21881 & 0.01018 & 703 & 0.21294 & -0.24727 & 0.78798 & -0.75369 \\
\hline 4 & -0.88494 & -0.00550 & -0.18563 & -0.05253 & 704 & 0.32029 & -0.27405 & 0.71352 & -0.77368 \\
\hline 5 & -0.85476 & -0.02788 & -0.31445 & -0.02149 & 705 & 0.34432 & -0.28957 & 0.70823 & -0.83357 \\
\hline 6 & -0.89577 & -0.11181 & -0.21073 & 0.01696 & 706 & 0.33246 & -0.40324 & 0.75217 & -0.67510 \\
\hline 7 & -0.85337 & -0.10144 & -0.31579 & 0.05071 & 707 & 0.34225 & -0.15256 & 0.77493 & -0.75078 \\
\hline 8 & -0.99127 & -0.02931 & -0.22439 & 0.05503 & 708 & 0.33916 & -0.34021 & 0.70677 & -0.67815 \\
\hline 9 & -0.96615 & -0.07045 & -0.15579 & 0.10155 & 709 & 0.2658 & -0.28443 & 0.63715 & -0.64778 \\
\hline 10 & -1.03280 & 0.080777 & -0.27235 & 0.03679 & 710 & 0.24144 & -0.3201 & 0.66818 & -0.73402 \\
\hline$\ldots$ & $\ldots$ & $\ldots$ & $\ldots$ & $\ldots$ & $\cdots$ & $\ldots$ & $\ldots$ & $\ldots$ & $\ldots$ \\
\hline 500 & -1.03050 & -0.03557 & -0.26615 & 0.00225 & 974 & 14.6570 & -1.51460 & 2.20830 & -2.2781 \\
\hline 501 & -1.00070 & -0.13426 & -0.29863 & -0.04624 & 975 & 26.6130 & 15.4070 & 3.11460 & -2.1513 \\
\hline 502 & -1.01900 & -0.11944 & -0.24604 & 0.00183 & 976 & 66.1990 & -19.1070 & 2.83770 & -0.52186 \\
\hline 503 & -0.97923 & -0.18848 & -0.29467 & -0.06398 & 977 & 33.8730 & -2.28540 & -0.24986 & -3.1073 \\
\hline 504 & -0.92120 & -0.12961 & -0.28593 & -0.01329 & 978 & 27.3770 & 9.77000 & -0.38371 & -1.3508 \\
\hline 505 & -0.99977 & -0.12605 & -0.25503 & -0.00760 & 979 & 14.1770 & -1.83210 & 2.93250 & 1.03660 \\
\hline 506 & -1.09560 & -0.11377 & -0.13928 & 0.03664 & 980 & 91.6100 & 5.00480 & -11.7640 & 0.95157 \\
\hline 507 & -0.99919 & -0.19812 & -0.20236 & 0.00973 & 981 & 27.1900 & -2.00060 & -0.69597 & 0.13394 \\
\hline 508 & -0.98075 & -0.14233 & -0.19172 & 0.03616 & 982 & 38.0490 & 1.90030 & 2.14970 & -0.32731 \\
\hline 509 & -1.03910 & -0.17238 & -0.19803 & 0.03396 & 983 & -2.10840 & -0.32350 & -0.73284 & -0.23275 \\
\hline 510 & -0.94198 & -0.02021 & -0.24582 & -0.02016 & 984 & -2.10840 & -0.22067 & -0.81421 & -0.36467 \\
\hline
\end{tabular}

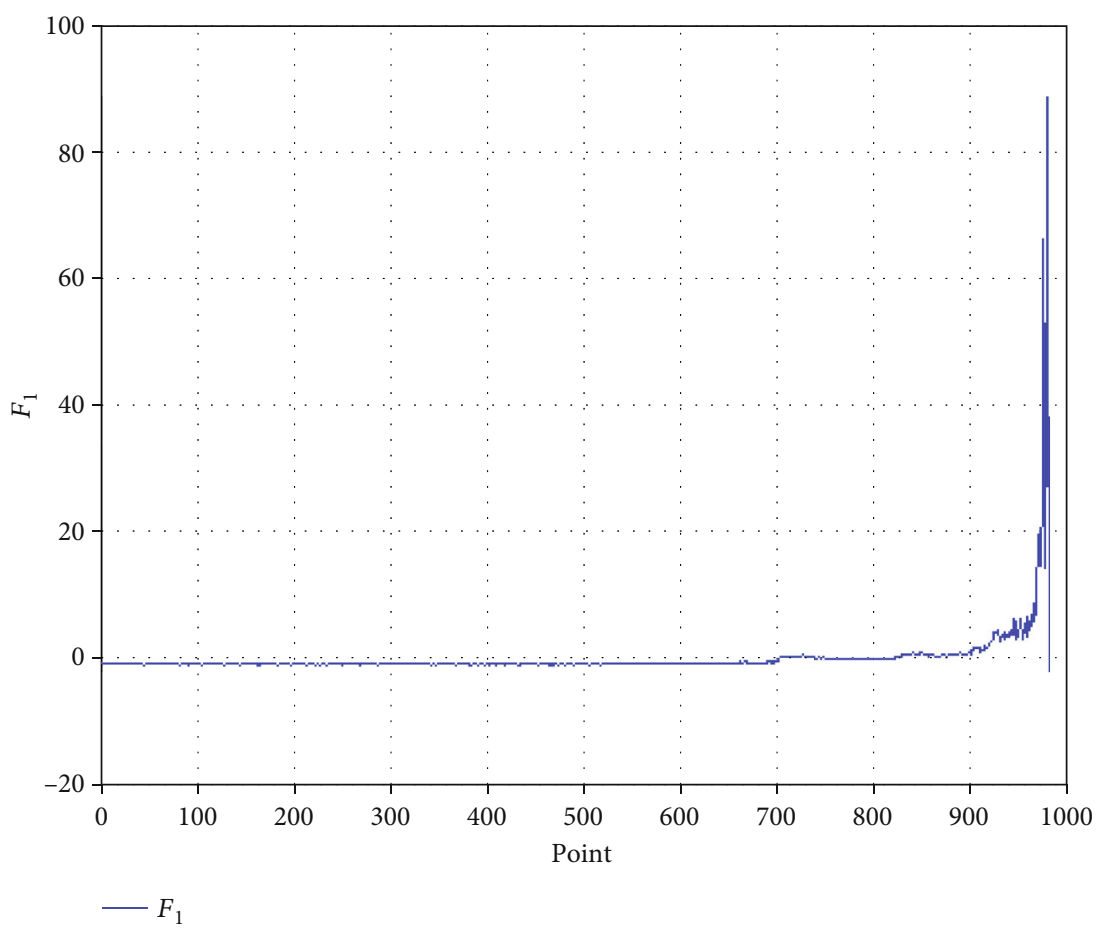

Figure 7: Performance declining curve of principal component $F_{1}$. 


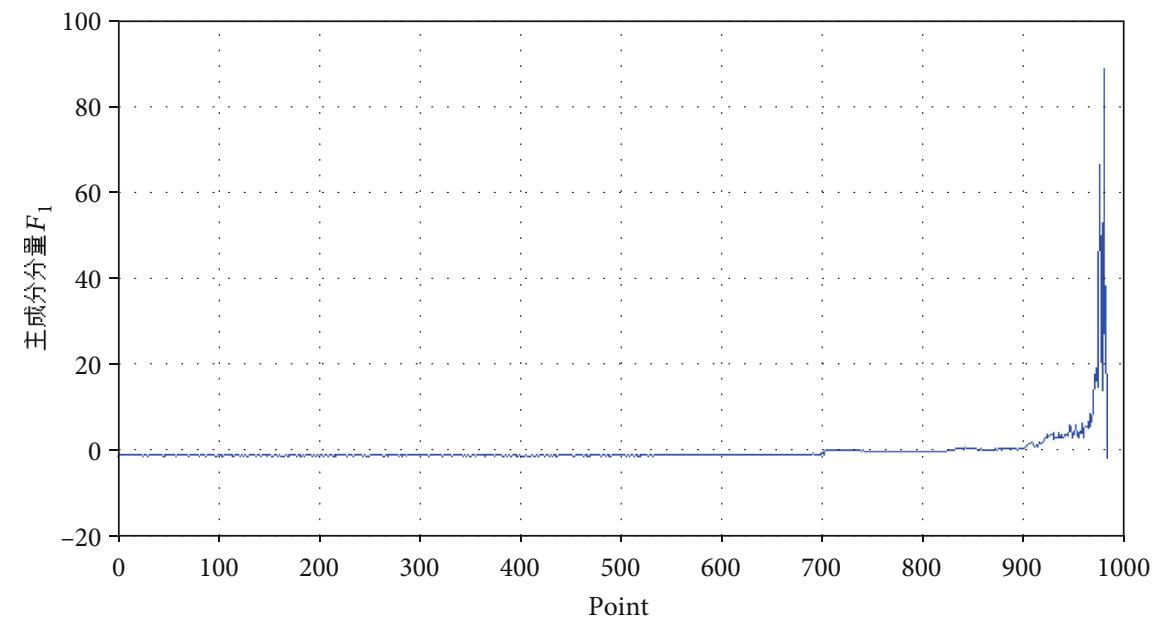

$-F_{1}$

(a)

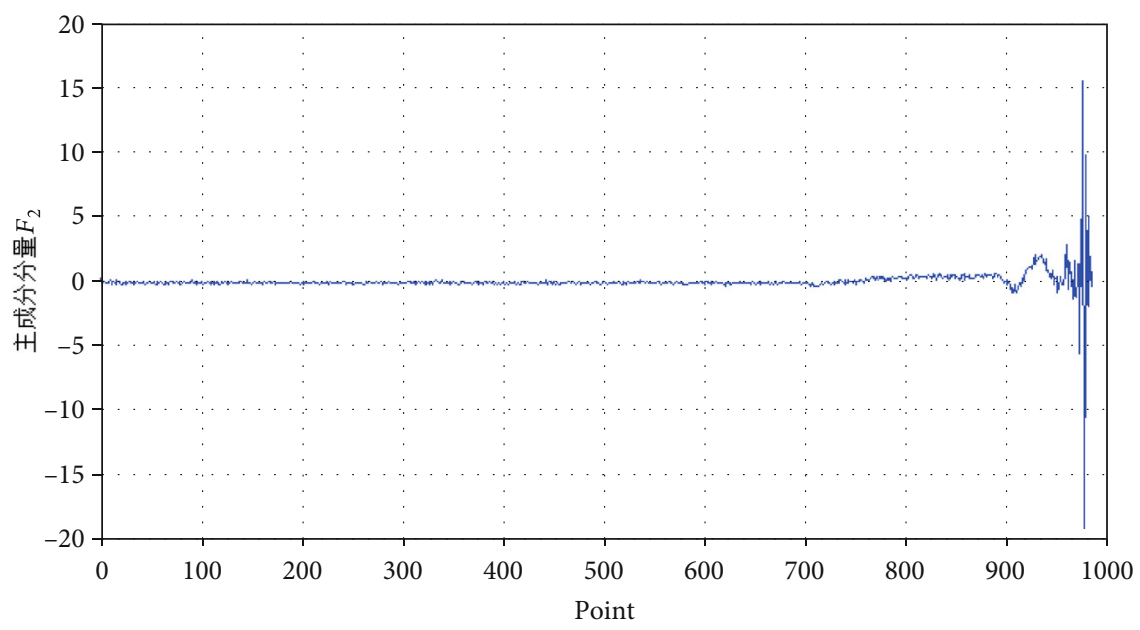

$-F_{2}$

(b)

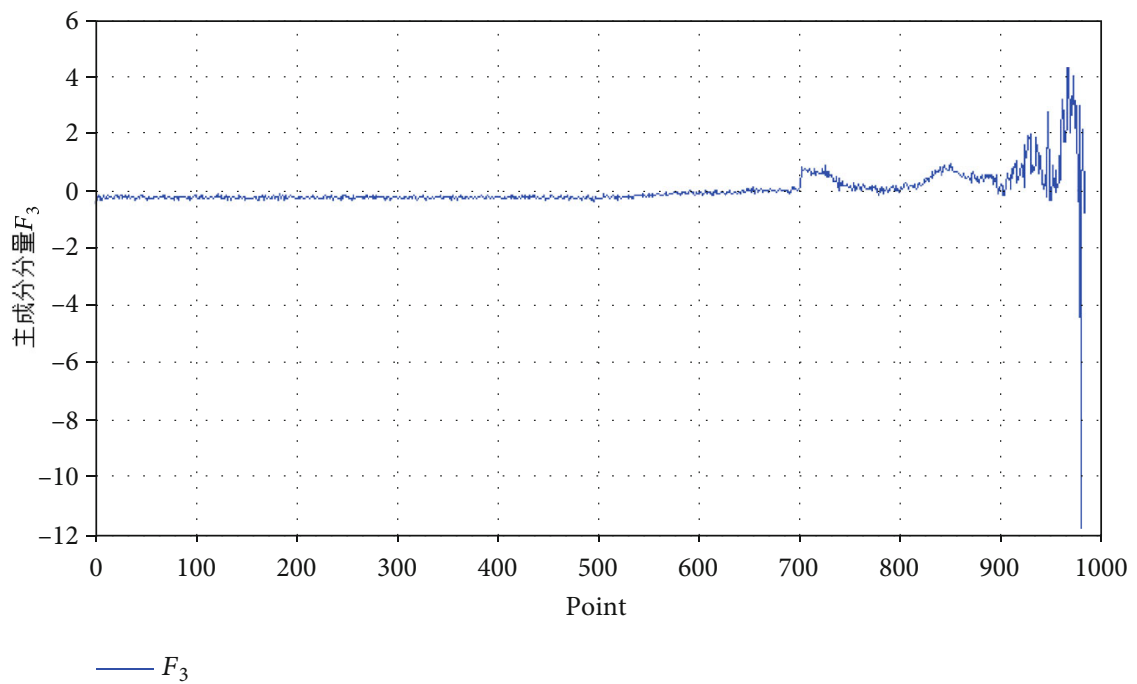

(c)

Figure 8: Continued. 


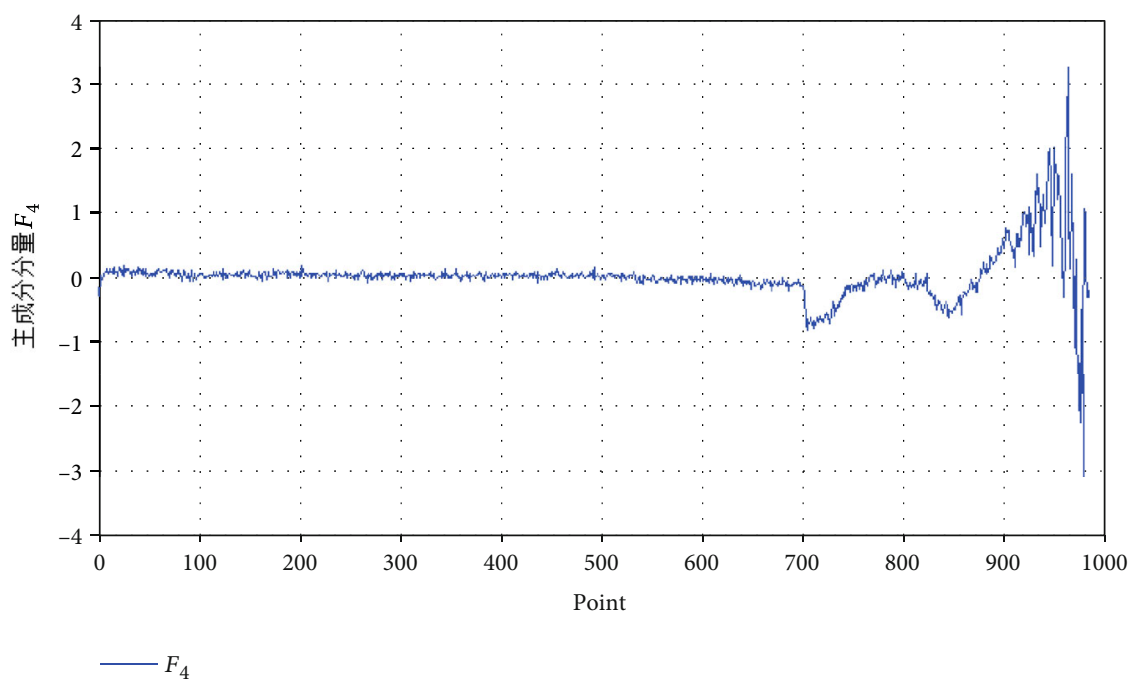

(d)

Figure 8: Performance decline curve of principal components $F_{1}, F_{2}, F_{3}$, and $F_{4}$.

In Figure 8, principal components $F_{1}$ and $F_{2}$ in Figures $8(\mathrm{a})$ and $8(\mathrm{~b})$ tend to be stable in the early stage (near the 500 sample points) and middle stage of performance degradation (near the 700 sample points) and does not show a significant upward trend until the late stage of performance degradation (near the 900 sample points). Principal components $F_{3}$ and $F_{4}$ in Figures $8(\mathrm{c})$ and $8(\mathrm{~d})$ tend to be stable in the early stage of performance degradation and increase slightly in the middle stage of performance degradation and does not show a significant upward trend until the late stage of performance degradation. Compared with the principal components $F_{1}$ and $F_{2}$, the changing trend of the principal components $F_{3}$ and $F_{4}$ is more obvious, which indicates that the feature information contained solely in the principal components $F_{1}$ and $F_{2}$ is not enough to characterize the decline of the performance of the $\mathrm{R} 2 \mathrm{R}$ processing roll for flexible material.

Considering the computational complexity in dimension reduction of principal component analysis data and the cumulative contribution rate of the eigenvalues $\lambda_{1}, \lambda_{2}$, and , $\lambda_{3}$ was 0.989 , which was much larger than the screening requirement of principal component analysis, three principal components $F_{1}, F_{2}$, and $F_{3}$ were selected to achieve the purpose of evaluating the performance degradation of R2R processing roll for flexible material. On the basis of this, a threedimensional contour map was drawn (see Figures 9(a) and 9(b)).

As can be seen from Figure 9(a), it is impossible to distinguish the decline process clearly because the amplitude of principal component feature index in the early stage is smaller and more concentrated, and the amplitude in late degradation stage is larger and more dispersed. For this reason, the red rectangular region in Figure 9(a) is enlarged locally as shown in Figure 9(b). The principal component values are clearly divided into three regions: the leftmost data centralized region, the middle narrow band region, and the right upper scatter region, which represents good performance and early degradation, medium-term degradation, and late degradation, respectively. Among them, the different colored contour lines on the left of Figure 9 realize the division between the good performance state (red area) and the early degradation state (purple area).

The experimental results showed that the principal components $F_{1}, F_{2}$, and $F_{3}$ of the vibration feature parameter of the $\mathrm{R} 2 \mathrm{R}$ processing roll for flexible material can not only distinguish the medium-term degradation and the late degradation state but also distinguish the normal state from the early degradation state, and the distinction is much more visualized. Therefore, it is further proved that the principal components $F_{1}, F_{2}$, and $F_{3}$ obtained by the method in the paper not only realizes data dimensionality reduction but also can characterize the performance degradation state of the $\mathrm{R} 2 \mathrm{R}$ processing roll for flexible material.

\section{Conclusion}

In this paper, a new principal component analysis method for data dimensionality reduction was proposed, to handle the problem of high-dimensional performance characterization and the complexity of calculating the performance indicators in flexible material roll-to-roll processing.

Based on the analysis of the performance influencing factors of flexible material roll-to-roll processing roller, a principal component analysis extraction model was constructed. And the research on the derivation of the extraction model algorithm was carried out.

The original feature parameter matrix composed of $10-$ dimensional feature parameters such as time domain, frequency domain, and time-frequency domain vibration signal of the roll shaft was established; then, we used the means of averaging to normalize the original feature parameters matrix. The correlation measure between every two parameters in the matrix was used as the eigenvalue to establish the covariance matrix of the performance degradation feature parameters. The Jacobi iteration method was introduced to derive the algorithm for solving eigenvalue and eigenvector 


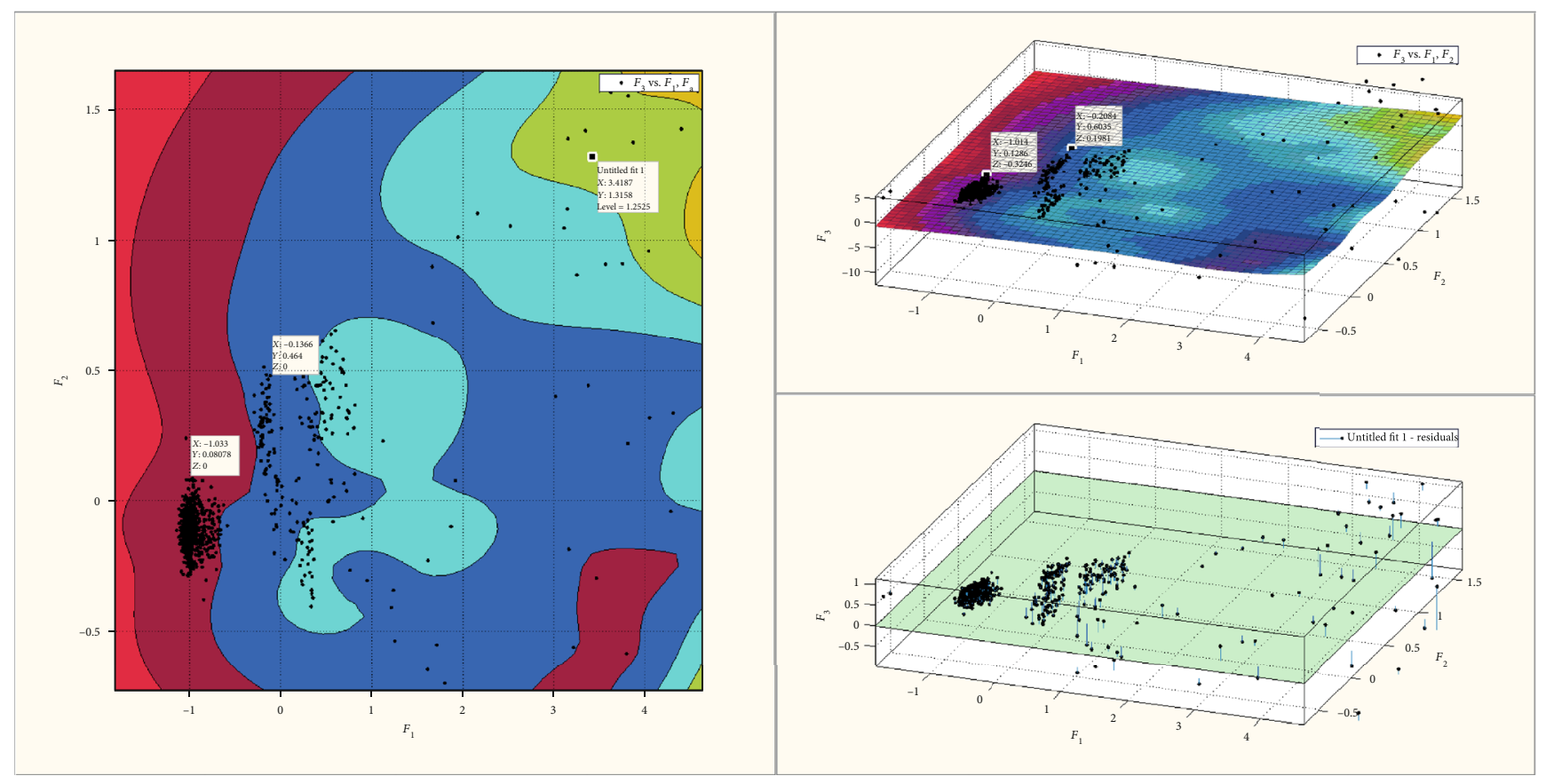

(a) Principal component three-dimensional global map
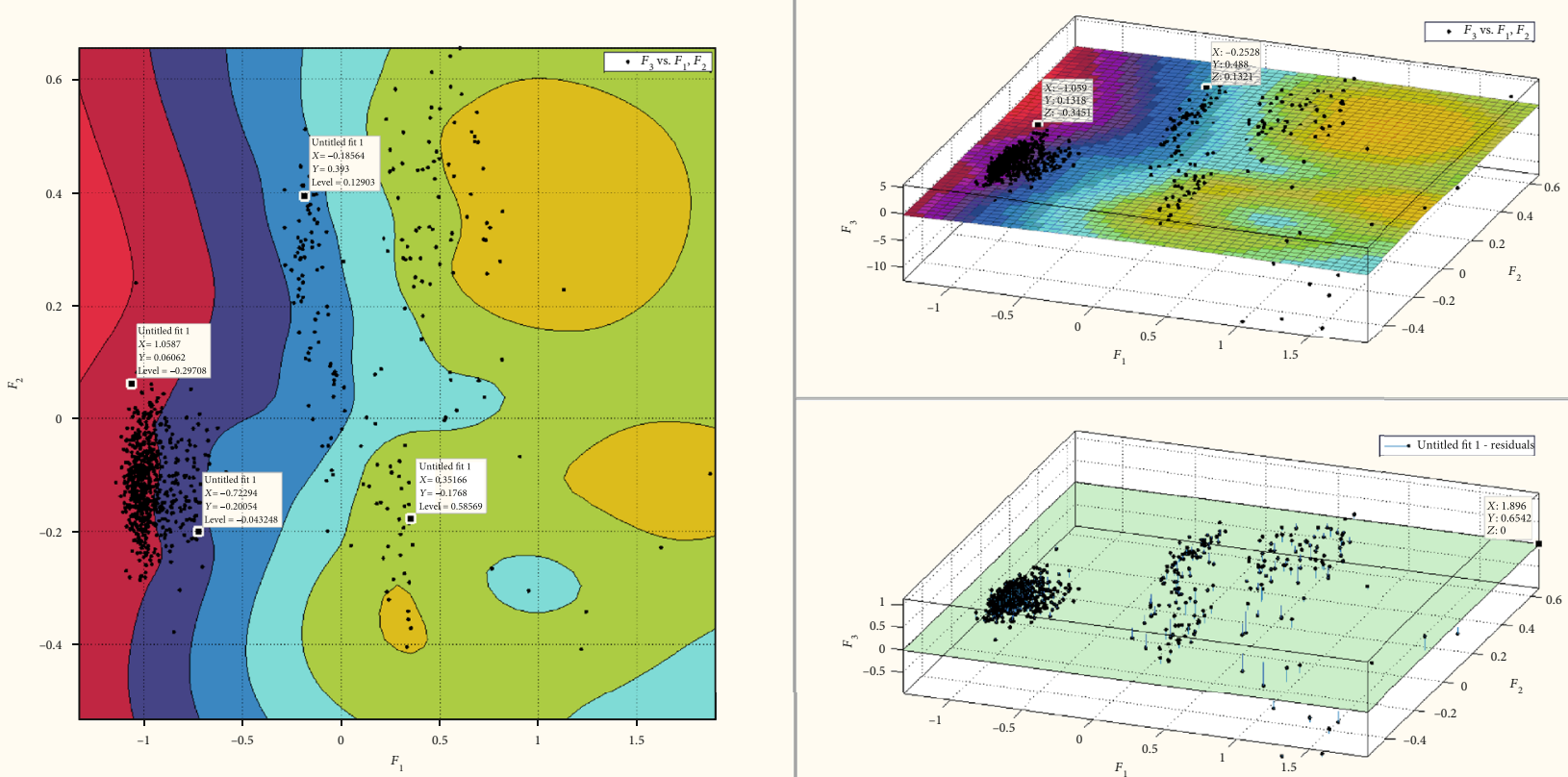

(b) Principal component three-dimensional spatial local map

FIgURE 9: Three-dimensional contour map of principal components $F_{1}, F_{2}$, and $F_{3}$.

of the covariance matrix. Finally, using the eigenvalue cumulative contribution rate as the screening rule, we linearly weighted and fused the eigenvectors and derived the feature principal component matrix $F$ of the processing roller vibration signal.

Experiments results showed that after the extraction by principal component analysis model, the initially obtained 10-dimensional features of the processing rollers' vibration signals, such as average, root mean square, kurtosis index, centroid frequency, root mean square of frequency, standard deviation of frequency, and energy of the intrinsic mode function component, can be expressed by 3-dimensional principal components $F_{1}, F_{2}$, and $F_{3}$. These three components contain $98.9 \%$ data information of the original vibration signal, which means that the feature parameter dimension reduction of processing roller vibration signal has been realized. The three-dimensional contour map drawn by $F_{1}, F_{2}$, and $F_{3}$ value not only can clearly distinguish the degraded state in the middle and late stages but also can monitor the state process of the roller from normal to 
early degradation and can also provide the roller predictive maintenance subsequent research with strong theoretical support.

The above experimental results show that the method in this paper has high accuracy in feature extraction of the performance degradation of flexible material R2R processing; it also has the advantages of eliminating the correlation between the feature variables and reducing the workload of feature selection. The research results of this paper can provide strong theoretical support for the follow-up predictive maintenance research of roll shaft.

\section{Data Availability}

The data that support the findings of this study are available from the corresponding author on request.

\section{Conflicts of Interest}

The authors declared no potential conflicts of interest with respect to the research, authorship, and/or publication of this article.

\section{Authors' Contributions}

Yaohua Deng designed the study, performed the research, analyzed the data, and wrote the paper. Huiqiao Zhou designed the study and performed the research. Kexing Yao analyzed the data and wrote the paper. Zhiqi Huang analyzed the data and contributed to the revision. Chengwang Guo contributed to the revision.

\section{Acknowledgments}

This work is supported in part by the National Natural Science Foundation of China under Grant No. 51675109 and in part by the Provincial Research and Development Project in Key Areas of Guangdong Province, China, under Grant No. 2019B010154001.

\section{References}

[1] Y. Deng, N. Zhou, X. Liu, and Q. Lu, "Research on fault diagnosis of flexible material R2R manufacturing system based on quality control chart and SoV," Mathematical Problems in Engineering, vol. 2018, Article ID 6350380, 8 pages, 2018.

[2] Y. Deng, Q. Lu, K. Yao, and N. Zhou, "Study on phosphor powder precipitation model in flexible material manufacturing process based on neuro-fuzzy network," Optik, vol. 168, pp. 563-576, 2018.

[3] Z. Shuai, "Laplacian eigenmaps and Mahalanobis distance based health assessment Methodology for Ball Screw," Journal of Mechanical Engineering, vol. 53, no. 15, pp. 125-130, 2017.

[4] J. Wu, "Data-driven performance degradation condition monitoring for rolling bearings," Journal of Shanghai Jiaotong University, vol. 52, no. 5, pp. 538-544, 2018.

[5] M. L. Fadda and A. Moussaoui, "Hybrid SOM-PCA method for modeling bearing faults detection and diagnosis," Journal of the Brazilian Society of Mechanical Sciences and Engineering, vol. 40, no. 5, pp. 1-8, 2018.
[6] Q. Mao, X. Fang, Y. Hu, and G. Li, "Chiller sensor fault detection based on empirical mode decomposition threshold denoising and principal component analysis," Applied Thermal Engineering, vol. 144, pp. 21-30, 2018.

[7] Z. Wu, "Rotating machinery early fault diagnosis method based on multivariate empirical mode decomposition," Chinese Journal of Scientific Instrument, vol. 37, no. 2, pp. 241248, 2016

[8] A. Tabrizi, L. Garibaldi, A. Fasana, and S. Marchesiello, "Early damage detection of roller bearings using wavelet packet decomposition, ensemble empirical mode decomposition and support vector machine," Meccanica, vol. 50, no. 3, pp. 865874, 2015.

[9] M. Kaleem, A. Guergachi, and S. Krishnan, "Hierarchical decomposition based on a variation of empirical mode decomposition," Signal Image and Video Processing, vol. 11, no. 5, pp. 793-800, 2017.

[10] J. Liu, Z. Xu, L. Zhou, Y. Nian, and Y. Shao, "A statistical feature investigation of the spalling propagation assessment for a ball bearing," Mechanism and Machine Theory, vol. 131, pp. 336-350, 2019.

[11] L. Da-wei, S. Hai-jiang, L. Wei-ning, and L. Pei-xun, "Improved sliding average target detection algorithm based on statistical information," Chinese Journal of Liquid Crystals And Displays, vol. 33, no. 6, pp. 497-503, 2018.

[12] Z. Long, "Assessment of rolling bearing performance degradation using gauss mixture model and multi-domain features," China Mechanical Engineering, vol. 25, no. 22, pp. 30663072, 2014.

[13] W. Jun, T. Lingyu, L. Yuyan, and G. Peng, "A weighted EMDbased prediction model based on TOPSIS and feed forward neural network for noised time series," Knowledge-Based Systems, vol. 132, pp. 167-178, 2017.

[14] J. Ou, Y. Li, and C. Shen, "Unlabeled PCA-shuffling initialization for convolutional neural networks," Applied Intelligence, vol. 48, no. 12, pp. 4565-4576, 2018.

[15] A. Enguix-González, J. M. Muñoz-Pichardo, J. L. MorenoRebollo, and I. Barranco-Chamorro, "Using conditional bias in principal component analysis for the evaluation of joint influence on the eigenvalues of the covariance matrix," Applied Mathematics and Computation, vol. 218, no. 17, pp. 89378950, 2012.

[16] A. Dürre, R. Fried, and D. Vogel, "The spatial sign covariance matrix and its application for robust correlation estimation," Austrian Journal of Statistics, vol. 46, no. 3-4, pp. 13-22, 2017.

[17] S. Camiz and V. Pillar, "Identifying the informational/signal dimension in principal component analysis.," Mathematics, vol. 6 , no. 11, p. $269,2018$.

[18] G.-H. Cheng, S.-M. Li, and E. Moreau, "New Jacobi-like algorithms for non-orthogonal joint diagonalization of Hermitian matrices," Signal Processing, vol. 128, pp. 440-448, 2016.

[19] S.-I. Tsukada, "Unbiased estimator for a covariance matrix under two-step monotone incomplete sample," Communications in Statistics-Theory and Methods, vol. 43, no. 8, pp. 1613-1629, 2014.

[20] L. Ye, "Elliptic function based algorithms to prove Jacobi theta function relations," Journal of Symbolic Computation, vol. 89, pp. 171-193, 2018.

[21] E. B. Kovač and V. Hari, "Jacobi method for symmetric $4 \times 4$ matrices converges for every cyclic pivot strategy," Numerical Algorithms, vol. 78, no. 3, pp. 701-720, 2018. 